\title{
Novitates
}

PUBLISHED BY THE AMERICAN MUSEUM OF NATURAL HISTORY CENTRAL PARK WEST AT 79TH STREET, NEW YORK, NY 10024

Number 3499, 31 pp., 94 figures November 29, 2005

\section{On the Chilean Spiders of the Family Prodidomidae (Araneae, Gnaphosoidea), with a Revision of the Genus Moreno Mello-Leitão}

\author{
NORMAN I. PLATNICK, ${ }^{1}$ MOHAMMAD U. SHADAB,${ }^{2}$ AND \\ LOUIS N. SORKIN ${ }^{3}$
}

\begin{abstract}
Although no members of the spider family Prodidomidae have previously been described from Chile, that country has a substantial, and largely endemic, prodidomid fauna. The Argentine genus Moreno Mello-Leitão is transferred from the Gnaphosidae to the Prodidomidae. Moreno was previously known only from the female holotype of its type species, but two additional species from Argentina and three from Chile are newly described, including the first known males of the genus. Three new genera are described: Chileomma, containing seven new six-eyed species from northern and central Chile; Chilongius, containing five new species from northern Chile; and Chileuma, containing three new species from northern and central Chile. A newly discovered feature on the tarsal claws is hypothesized to be a synapomorphy uniting Moreno and Chilongius with the other South American tricongiine Theuminae.
\end{abstract}

\section{INTRODUCTION}

The spider family Prodidomidae is widespread, occurring on all continents except Antarctica, but is much more diverse in the southern hemisphere than in Laurasian areas. In South America, it is represented by such genera as Prodidomus Hentz (see Dalmas, 1919, and Cooke, 1964), Zimiris Simon (see

\footnotetext{
${ }^{1}$ Peter J. Solomon Family Curator, Division of Invertebrate Zoology, American Museum of Natural History; Adjunct Professor, Department of Biology, City College, City University of New York; Adjunct Professor, Department of Entomology, Cornell University; Adjunct Senior Research Scientist, Center for Environmental Research and Conservation, Columbia University (platnick@amnh.org).

${ }^{2}$ Senior Scientific Assistant, Division of Invertebrate Zoology, American Museum of Natural History.

${ }^{3}$ Senior Scientific Assistant, Division of Invertebrate Zoology, American Museum of Natural History.
} 

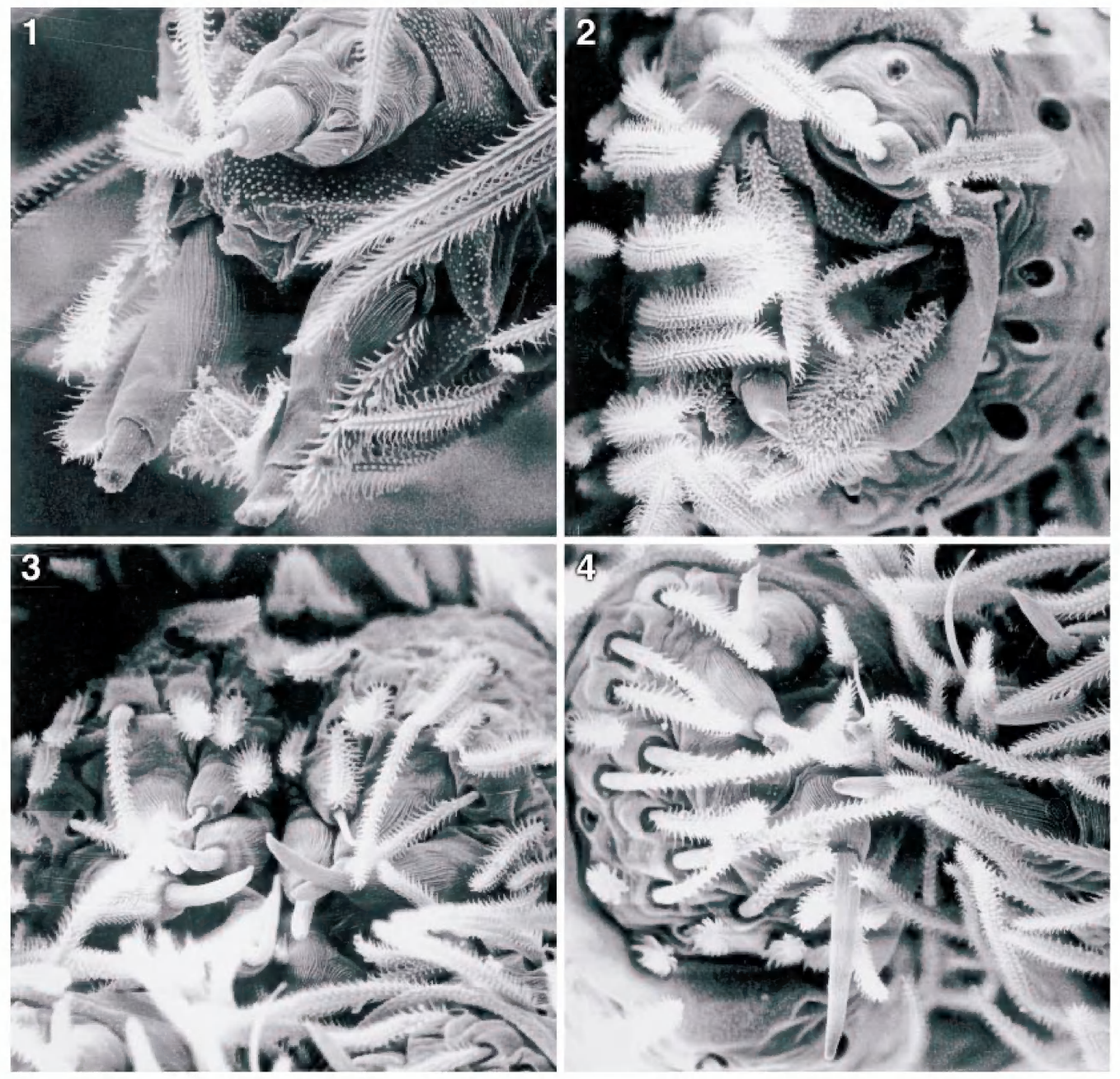

Figs. 1-4. Moreno chivato, new species. 1. Juvenile, anterior lateral spinnerets, distal view. 2. Female, same. 3. Female, posterior median spinnerets, distal view. 4. Female, posterior lateral spinnerets, distal view.

Platnick and Penney, 2004), Neozimiris Simon (see Platnick and Shadab, 1976), Lygromma Simon (see Platnick and Shadab, 1976), Tricongius Simon (see Platnick and Höfer, 1990), and Oltacloea Mello-Leitão (see Platnick, 1986).

Although no prodidomids have been described from Chile, a few specimens that seemed to belong to the family were found in Chilean collections made in the 1950's and 1960's by two Californian entomologists, Drs. Edward Ross and Evert Schlinger, and by the North American arachnologist, Dr. Allan Archer. Over the past 25 years, the first author and various colleagues have made several collecting trips to Chile, in search of prodidomids as well as other taxa, and we report here on the results of those expeditions.

With this new material in hand, it is clear that Chile actually harbors a substantial prodidomid fauna, including representatives of at least five genera. One is the widespread genus Prodidomus, which appears to be rep- 
resented in Chile only by its synanthropic type species, P. rufus Hentz. That species, and its distribution and synonyms, are discussed in detail in Platnick and Baehr (in press), and our focus here is instead on the endemic Chilean members of the family.

Those taxa belong not to the Prodidominae but to a group that has often mistakenly been referred to as the Anagraphidinae; as detailed in Platnick and Baehr (in press), the Old World type genus of that subfamilial name is misplaced in the Prodidomidae, and the New World "anagraphidines" (i.e., Lygromma, Tricongius, and Oltacloea) actually belong to the subfamily Theuminae.

As is typically the case for spider groups that have speciated within Chile, the theumine fauna seems to be entirely distinct, at the generic level, from that found in tropical South America, but does show some transAndean connections with Argentine taxa. In this case, one of the four genera discussed below is known to occur also in Argentina, where a female of one species was described (in a monotypic genus in the Gnaphosidae) as Moreno morenoi by Mello-Leitão (1940). The relationships and placement of Moreno are discussed in detail below.

Specimens have been examined from the collections of the American Museum of Natural History (AMNH), California Academy of Sciences (CAS), Museo Argentino de Ciencias Naturales (MACN), and Museo de La Plata (MLP). All measurements are in millimeters; epigyna were examined in clove oil to allow detailed tracing of their elaborate ducts. Chilean locality records are ordered geographically, by regions and provinces, arranged from north to south.

\section{SYSTEMATICS}

\section{FAMILY PRODIDOMIDAE}

DiAGNOSIS: Prodidomids are "higher gnaphosoids", resembling the Lamponidae and Gnaphosidae (rather than the Ammoxenidae, Cithaeronidae, Gallieniellidae, or Trochanteriidae) in having the anterior lateral spinnerets composed of a single article. In the "lower gnaphosoid" families, a separate distal segment is represented by a complete sclerotized ring surrounding the spigots on the anterior lateral spinnerets. The loss of the distal scler- otized ring enables the higher gnaphosoids to expand the soft cuticle surrounding those spigots. Prodidomids differ from lamponids in having enlarged piriform gland spigots that are much larger than the major ampullate gland spigots, and from true gnaphosids in having those piriform gland spigots greatly elongated (with long bases bearing short shafts) rather than widened, and accompanied at their base by long, plumose setae (Platnick, 1990, 2000, 2002).

\section{Key to Subfamilies and Genera of Chilean Prodidomidae}

1. Posterior eye row very strongly procurved, so much so that the eyes of each side appear to form a single row together with the anterior lateral eyes; posterior lateral spinnerets greatly enlarged, much larger than other

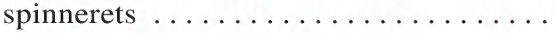
. Prodidominae, Prodidomus rufus Hentz

- Posterior eye row normal, not so strongly procurved; posterior lateral spinnerets no larger than anterior lateral spinnerets .... ............... Theuminae, 2

2. Abdomen with clavate setae (fig. 31) ..... ....................... Moreno

- Abdomen without clavate setae .......3

3. Six eyes ............. Chileomma

- Eight eyes ...............4

4. Tarsal claws dentate ....... Chilongius

- Tarsal claws smooth ........ Chileuma

\section{Moreno Mello-Leitão}

Moreno Mello-Leitão, 1940: 47 (type species by original designation Moreno morenoi MelloLeitão, 1940).

Diagnosis: Specimens of Moreno can easily be distinguished from those of the other New World prodidomid genera by the presence of large, clavate, translucent setae on the abdominal dorsum (fig. 31; Mello-Leitão, 1940, fig. 49).

Placement: Mello-Leitão (1940) described Moreno as a member of the Gnaphosidae, but placed it only within the illdefined "Drassoidinarum", and Roewer (1955) retained the genus within the Drassodinae by default. However, the morphology of the piriform gland spigots on the anterior lateral spinnerets indicates that these spiders are prodidomids rather than gnaphosids. Although few in number, the piriform 
gland spigots are elongated rather than widened, with long bases and short shafts, and are accompanied by at least one long seta at their base (figs. 1, 2). Within the Prodidomidae, the morphology of both the sternum and the epigynum supports the placement of the genus within the Theuminae. The sternum has a large protrusion between the fourth coxae that lacks the rebordered margin found on the sternal sides but bears numerous long, erect setae; the epigynum has highly convoluted ducts.

RELATIONShiPs: The tarsal claws of Moreno are unusual, in that they bear both a series of normal teeth and a set of closely spaced teeth situated at the base of each claw (fig. 5 ). These closely spaced teeth are strongly arched, and are apparently used to grasp a short, specialized seta that originates just dorsally of the much longer claw tuft setae (fig. 6). Scanning electron microscopy of the claws of representatives of the new genera Chileomma and Chileuma, as well as of Theuma, Lygromma, and Tivodrassus, has revealed no similar specializations, and Dr. Barbara Baehr has confirmed (in litt.) that they also do not occur in any of the Australian molycriine genera. However, apparently homologous basal teeth do occur on the claws of Chilongius (figs. 7, 8) and an undescribed Argentine prodidomid that appears to be closest to Tricongius (figs. 9, 10). Although we have no specimens of Tricongius available for scanning, we hypothesize that these specialized teeth and setae will be found to occur in that genus as well, and that the character will prove to be synapomorphic for this set of taxa. If that hypothesis is correct, the tribal name Tricongiini, based on $\mathrm{Si}$ mon's (1893) group Tricongieae, would be available for this group of genera.

DESCRIPTION: Small spiders, total length 1.8-2.7. Carapace oval, narrowed in front to half its maximum width, with only anterior margin heavily sclerotized, posterior margin invaginated at pedicel, not reflexed; surface coated with recumbent, light setae, without tubercles; thoracic groove short, shallow, longitudinal. Eight subequal eyes in two rows, anterior medians circular, dark, other eyes oval, light, posterior medians flat, irregularly rectangular; from above, anterior eye row recurved, posterior row slightly re- curved, from front, anterior row recurved, posterior row slightly procurved; anterior medians separated by slightly more than their diameter, almost touching anterior laterals; posterior medians separated by about their maximum width, almost touching posterior laterals; anterior and posterior laterals separated by less than their radius; median ocular quadrangle wider in back than in front, about as long as wide in back. Chelicerae vertical, not divergent, paturon without boss, promargin with row of long, curved setae, seta closest to fang base greatly elongated, distinctly bent toward midline at about one-eighth its length; promargin with three teeth, retromargin with two denticles, fang short, not arched; chilum indistinct, apparently unipartite, triangular. Labium wider than long, widest posteriorly, along procurved posterior margin; anterior margin strongly rebordered. Endites short, convergent anteriorly, bent and obliquely depressed near anterior margin of labium; serrula apparently absent (but absence not confirmed by scanning electron microscopy). Sternum shield-shaped, flat, with strongly rebordered lateral margins but rebordering abruptly absent along distinct posterior protrusion between coxae IV, not expanded anteriorly, with only indistinct extensions between coxae but with large, triangular extensions to middle of coxae; surface smooth, with long setae, posterior protrusion with numerous long, erect setae, widely separating coxae IV. Single epimeric sclerite on each side, above coxae, not extending between coxae, not fused to carapace. Pedicel composed of two dorsal sclerites (both sclerites excavated anteriorly) and sclerotized, triangular ventral sclerite almost reaching posterior tip of sternum.

Abdominal dorsum without anterior scutum in either sex; cuticle with long, recumbent setae, dorsum also with large, clavate, translucent setae (fig. 31); epigastric scutum weakly sclerotized, without postepigastric sclerites, booklung covers not ridged; colulus represented by paired clusters of setae originating about halfway between anterior lateral spinnerets and wide, straight posterior spiracle. Six spinnerets, anterior laterals moderately elongated, widely separated, not advanced anteriorly, with elongate ventral tubercle arising near cuticle of basal segment, 

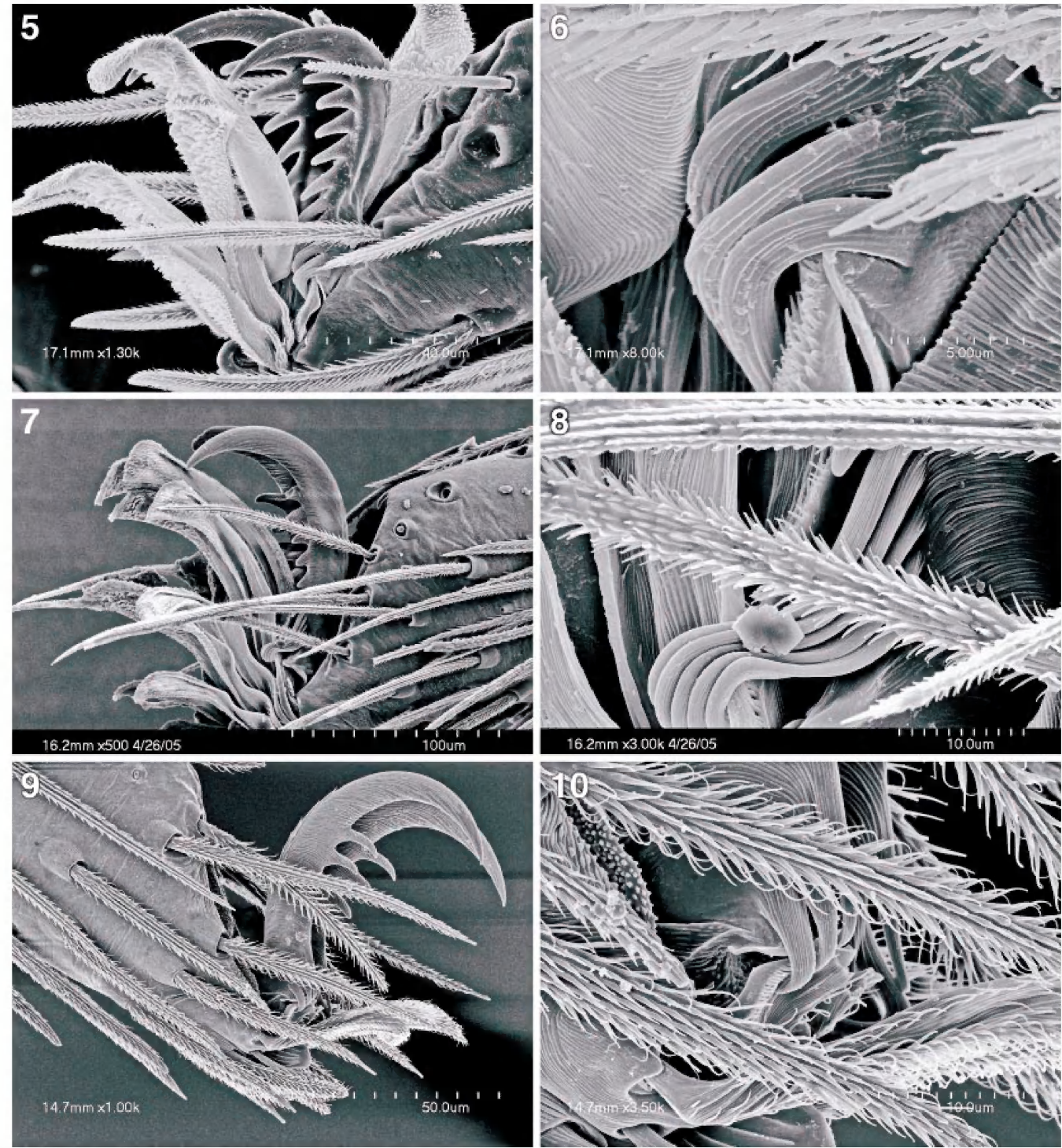

Figs. 5-10. 5, 6. Moreno chivato, new species. 7, 8. Chilongius palmas, new species. 9, 10. Undescribed prodidomid from Iguazú, Misiones, Argentina. 5, 7, 9. Tarsus IV of female, lateral view, showing normal, distal claw teeth and specialized, proximal claw teeth. 6, 8, 10. Specialized claw teeth and the specialized seta they interlock with.

bearing major ampullate gland spigots (figs. $1,2)$, soft portions of cuticle bearing few piriform gland spigots with elongated bases, short shafts, and at least one accompanying seta (figs. 1, 2); female posterior medians small, narrow, contiguous, with spigots closely clustered at tip, cluster apparently consisting of one large cylindrical gland spigot, one minor ampullate gland spigot with narrow shaft, and two or three aciniform gland spigots (fig. 3); female posterior laterals apparently with one large cylindrical 
gland spigot, one minor ampullate gland spigot with narrow shaft, and two aciniform gland spigots (fig. 4).

Leg formula 4123, legs short, coated with erect, dark setae; coxae and trochanters without dorsal tubercles, fourth trochanters only slightly elongated; anterior coxae without protuberant posterolateral corners; trochanters not notched; metatarsi and tarsi without scopula; posterior metatarsi without distal preening brushes; tarsi without cuticular cracks, with two long claws bearing normal teeth and more proximal set of closely spaced, proximally directed teeth, most proximal of which clasps base of modified seta (figs. 5, 6), claw tufts composed of few, distally widened setae; dorsal surface of tarsi with unmodified proximal margin; trichobothria apparently few, in two rows dorsally on all tarsi and metatarsi. Female palpal femur, patella, tibia, and tarsus with several short spines, tarsus short, not enlarged distally, without distodorsal pad of setae, apparently without claw. Leg spines of males longer than those of females; typical leg spination pattern (only surfaces bearing spines listed): femora: I, II d1-1-0, p0-0-1; III, IV d1-1-0, p0-0-1, r0-0-1; tibiae: I, II v2-2-0; III, IV d1-0-0, p0-1-1, v2-2-2, r0-1-1; metatarsi: I, II v2-0-0; III, IV p0-1-2, v2-0-0, r0$1-2$.

Male palpal tibia with bifid retrolateral apophysis, ventral prong much shorter than dorsal prong; embolus short, originating prodistally, accompanied by transparent conductor (often difficult to observe, unless palp is at least partially expanded); median apophysis distinctly twisted. Epigynum with conspicuous, posterior openings; ducts highly convoluted.

\section{Moreno morenoi Mello-Leitão}

Figures 11, 12

Moreno morenoi Mello-Leitão, 1940: 47, figs. 46-49 (female holotype from Tandil, Buenos Aires, Argentina, in MLP, examined).

DiAGNOSIS: Males are unknown; females have large, sausage-shaped lateral epigynal ducts (figs. 11, 12).

MALE: Unknown.

FEMAlE: Total length 2.62. Carapace 1.01 long, 0.79 wide. Femur II 0.66 long. Cara- pace and mouthparts light yellow except for black patch surrounding anterior median eyes, sternum paler, abdomen white, legs light yellow. Leg spination: femora: II p0-00; III p0-0-0, r0-0-0; IV p0-0-0; tibiae: I v44-2; II v0-4-2; III d1-1-0, p0-0-1, v0-1p-1p, r0-0-1; IV d1-1-0, p0-0-1, v0-1p-2, r0-0-1; metatarsi: I v2-2-2; II v2-1p-1p; III p0-0-1, v0-0-0, r0-0-1; IV p0-0-0, v0-1p-2, r0-0-0. Epigynal openings circular, situated anterior of laterally divided median septum (fig. 11); lateral ducts large, sausage-shaped (fig. 12).

Material ExAmined: ARGENTINA: Buenos Aires: Cerro Negro, Sierra de La Ventana, Sept. 29-Oct. 2, 1972 (M. Galiano, MACN), 1 \% ; Tandil, Dec. 1938 (M. Birabén, MLP 14.365), 19 (holotype).

Distribution: Known only from the Sierra de La Ventana in Buenos Aires province, Argentina; these mountains are also the home of the only known South American mecicobothriid spider, Mecicobothrium thorelli Holmberg (see Gertsch and Platnick, 1979).

\section{Moreno ramirezi, new species Figures 15-18}

TyPe: Male holotype from Refugio CaaPorá, Parque Provincial Uruguaí, $3 \mathrm{~km} \mathrm{~W}$ Deseado, Misiones, Argentina (Feb. 14-15, 1995; M. Ramírez), deposited in MACN.

ETyMOLOGY: The specific name is a patronym in honor of the collector of all the known specimens of this species, Dr. Martín Ramírez.

Diagnosis: Males can be recognized by the relatively short dorsal prong on the retrolateral tibial apophysis, the deep notch between the dorsal and ventral prongs on that apophysis, and the long-necked median apophysis (figs. 15, 16), females by the narrow anterior loops of the epigynal ducts (figs. 17, 18).

Male: Total length 1.80. Carapace 0.88 long, 0.65 wide. Femur II 0.58 long. Coloration as in $M$. morenoi. Leg spination: femora: II p0-0-0; III, IV p0-0-0, r0-0-0; tibiae: I v4-4-2; II v2-2-2; III d0-0-0, p0-0-1, v01p-2, r0-0-1; IV d0-0-0, p0-0-1, v0-2-2; metatarsi: I v2-2-2; II v2-2-0; III p0-0-1, v20-2, r0-0-1; IV p0-0-1, r0-0-1. Retrolateral tibial apophysis with relatively short dorsal prong separated from ventral prong by deep 

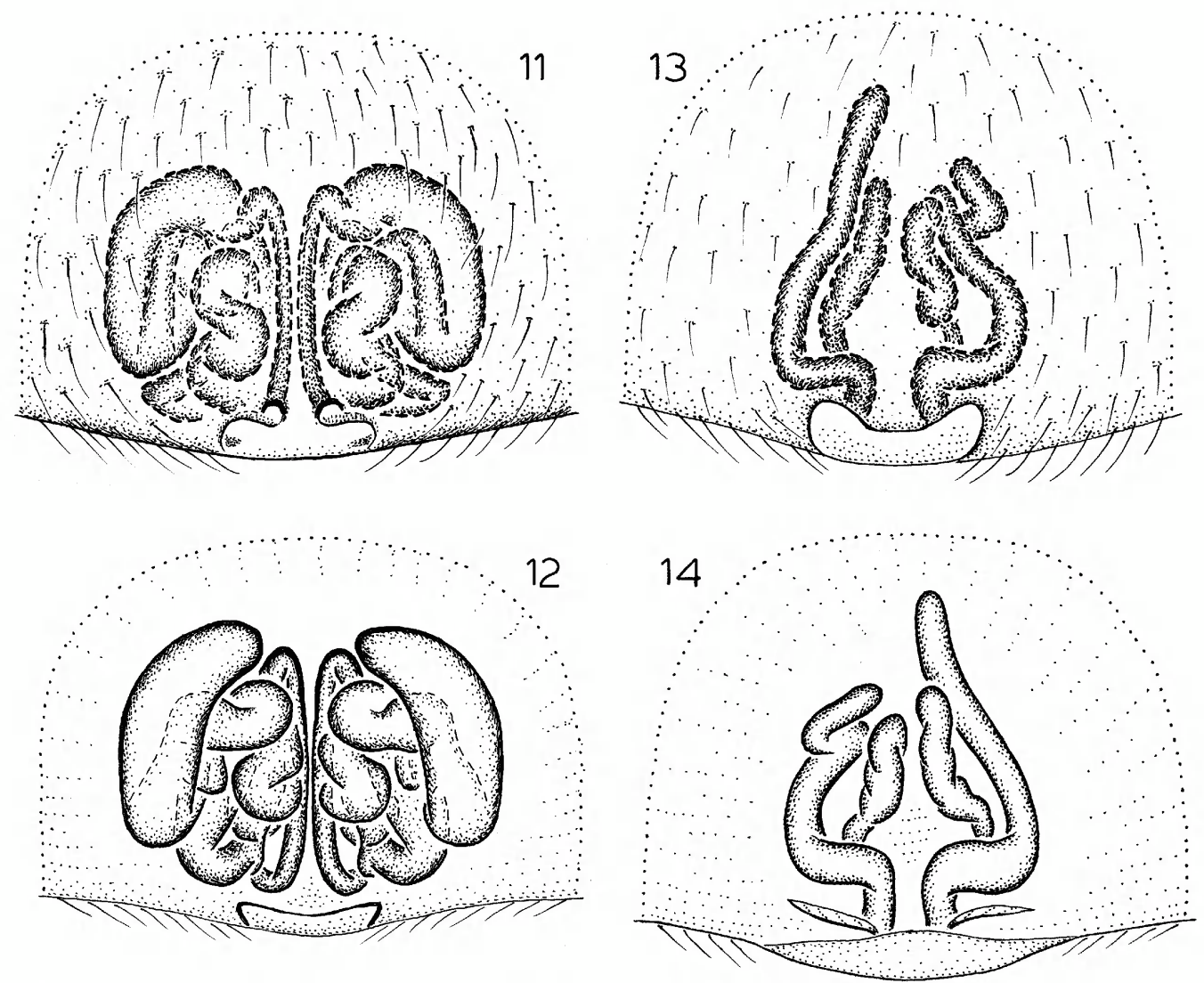

Figs. 11-14 11, 12. Moreno morenoi Mello-Leitão, female epigynum. 13, 14. Moreno neuquen, new species, same. 11, 13. Ventral view. 12, 14. Dorsal view.

notch (fig. 16); median apophysis relatively long, with long neck (fig. 15).

FEMALE: Total length 2.25. Carapace 0.95 long, 0.68 wide. Femur II 0.64 long. Coloration as in $M$. morenoi. Leg spination: femora: I, II p0-0-0; III, IV p0-0-0, r0-0-0; tibiae: I v4-4-0; II 2-4-0; III, IV d0-0-0, p0-0-1, v02-2, r0-0-1; metatarsi: I, II v2-2-2; III, IV p00-1, v1p-0-0, r0-0-1. Epigynal ducts relatively narrow, especially along anterior loop (figs. 17, 18).

OTHER MATERIAL EXAMINED: ARGENTINA: Misiones: INTA San Vicente, $10 \mathrm{~km} \mathrm{~N}$ San Vicente, Jan. 26, 1997 (M. Ramírez, MACN), $1 q$; Parque Nacional Iguazú, Oct. 23-26, 1995, Berlese (M. Ramírez, MACN), 1ठ; Parque Provincial Cruce Caballero, NE
San Pedro, Oct. 27-29, 1995 (M. Ramírez, MACN), $1 \hat{\sigma}$.

Distribution: Known only from Misiones, Argentina.

Moreno neuquen, new species Figures 13, 14

TyPE: Female holotype from Neuquén, Argentina (Sept. 1973; Ferrarris), deposited in MACN.

ETYMOLOGY: The specific name is a noun in apposition taken from the type locality.

DiAGNOSIS: Males are unknown, but females can be recognized by relatively simple epigynal ducts (figs. 13, 14).

MALE: Unknown.

Female: Total length 2.66. Carapace 0.91 

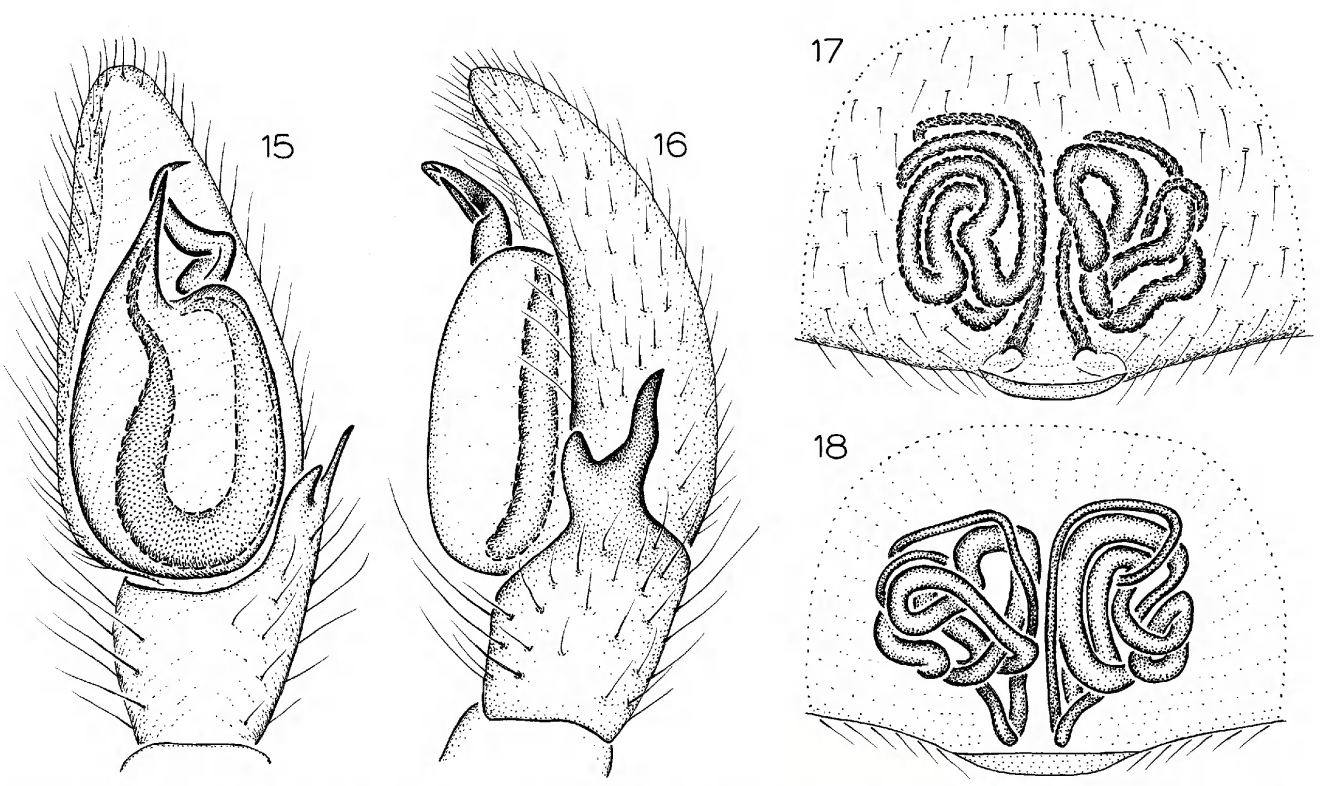

Figs. 15-18. Moreno ramirezi, new species. 15. Left male palp, ventral view. 16. Same, retrolateral view. 17. Epigynum, ventral view. 18. Same, dorsal view.

long, 0.73 wide. Femur II 0.57 long. Coloration as in $M$. morenoi. Leg spination: femora: I d1-0-0, p0-0-0; II p0-0-0; III, IV p0$0-0$, r0-0-0; tibiae: III, IV d0-0-0, p0-0-1, v02-2, r0-0-1; metatarsi: III, IV p0-0-1, r0-0-1. Epigynal ducts relatively simple, with only median and paramedian components (figs. 13,14 ; although it is possible that lateral components have been lost in preparation, this does not appear to be the case).

Other MATERIAl Examined: None.

Distribution: Known only from Neuquén, Argentina.

\section{Moreno chacabuco, new species} Figures 19-22

TYPE: Female holotype taken at an elevation of $1235 \mathrm{~m}$ on a dry mountainside with acacias on the north side of Cuesta de Chacabuco, Chacabuco, Región Metropolitana de Santiago, Chile (Jan. 14, 1985; N. Platnick, O. Francke), deposited in AMNH.

ETYMOLOGY: The specific name is a noun in apposition taken from the type locality.

DiAGNOSIS: Males resemble those of $M$. chivato in having only a shallow notch between the dorsal and ventral prongs of the retrolateral tibial apophysis (fig. 20), but differ in having a relatively short, short-necked median apophysis (fig. 19); females also resemble those of $M$. chivato in the shape of the posterior epigynal septum and openings (fig. 21) but differ in having longer, dorsally elevated median ducts (fig. 22).

MaLE: Total length 1.94. Carapace 0.92 long, 0.71 wide. Femur II 0.59 long. Coloration as in M. morenoi. Leg spination: femora: I, II p0-0-0; III, IV p0-0-0, r0-0-0; tibiae: III, IV p0-0-1, v2-0-2; metatarsi: I, II v2-40 ; III, IV v1p-0-0. Retrolateral tibial apophysis with short ventral prong bearing distal denticle, shallowly separated from long dorsal prong (fig. 20); median apophysis relatively short, with short neck (fig. 19).

Female: Total length 2.65. Carapace 0.92 long, 0.73 wide. Femur II 0.76 long. Coloration as in M. morenoi. Leg spination: femora: I, II p0-0-0; III, IV p0-0-0, r0-0-0; tibiae: I v4-4-0; metatarsi: I v4-4-2; II v4-4-0. Median epigynal ducts relatively long (fig. 21), dorsally elevated for about half their length (fig. 22).

Other Material Examined: CHILE: Región de Valparaíso (V): San Felipe de 

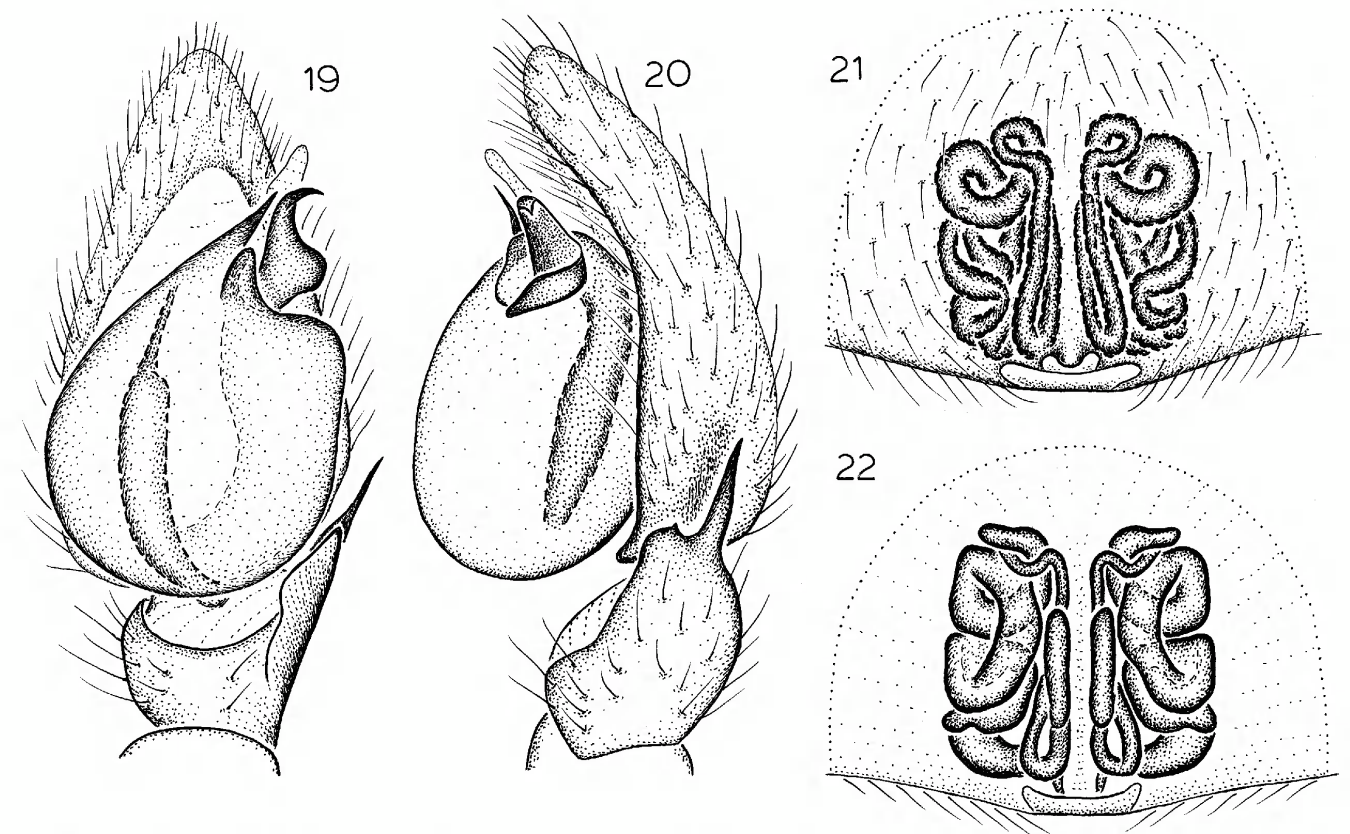

Figs. 19-22. Moreno chacabuco, new species. 19. Left male palp, ventral view. 20. Same, retrolateral view. 21. Epigynum, ventral view. 22. Same, dorsal view.

Aconcagua: Palmas de Ocoa, Parque Nacional La Campana, May 17, 1985, pitfalls, burned site (R. Calderón, AMNH), $2 \hat{0}$.

DisTRIBUTION: Known only from central Chile (regions V and Santiago).

\section{Moreno chivato, new species Figures 1-6, 23-26, 31}

TYPE: Female holotype taken at an elevation of $10 \mathrm{~m}$ at Quebrada del Chivato, $1 \mathrm{~km}$ S Los Molles, Petorca, Región de Valparaíso (V), Chile (Oct. 2, 1992; N. Platnick, P. Goloboff, K. Catley), deposited in AMNH.

ETYMology: The specific name is a noun in apposition taken from the type locality.

DiAgNosis: Males resemble those of $M$. chacabuco in having only a shallow notch between the dorsal and ventral prongs of the retrolateral tibial apophysis (fig. 24), but differ in having a relatively long, long-necked median apophysis (fig. 23); females also resemble those of $M$. chacabuco in the shape of the posterior epigynal septum and openings (fig. 25) but differ in having shorter, dorsally unelevated median ducts (fig. 26).
Male: Total length 2.18. Carapace 0.88 long, 0.66 wide. Femur II 0.58 long. Coloration as in $M$. morenoi. Leg spination: femora: II p0-0-0; IV p0-0-0; tibiae: I, II v0-1r0 ; III d0-0-0, p0-0-1, v0-1p-0, r0-0-1; IV v1p-1p-2; metatarsi: III, IV p0-0-1, r0-0-1. Retrolateral tibial apophysis with short ventral prong separated from long dorsal prong only by ledge (fig. 24); median apophysis relatively long, with long neck (fig. 23).

FEMALE: Total length 2.64. Carapace 1.10 long, 0.87 wide. Femur II 0.65 long. Coloration as in $M$. morenoi. Leg spination: femora: II p0-0-0; III, IV p0-0-0, r0-0-0; tibiae: I v2-4-2; II v0-1r-0; III d0-0-0, v0-2-2, r0-10 ; IV v0-2-2; metatarsi: I, II v2-4-0; III p00-1, r0-0-1; IV p0-0-2, r0-0-1. Median epigynal ducts relatively short (fig. 25), not dorsally elevated (fig. 26).

Other Material Examined: CHILE: Región de Valparaíso (V): Petorca: Quebrada del Chivato, $1 \mathrm{~km} \mathrm{~S}$ Los Molles, Oct. 30, 1988 (P. Goloboff, E. Maury, C. Szumik,

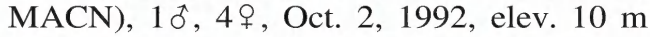
(N. Platnick, P. Goloboff, K. Catley, 

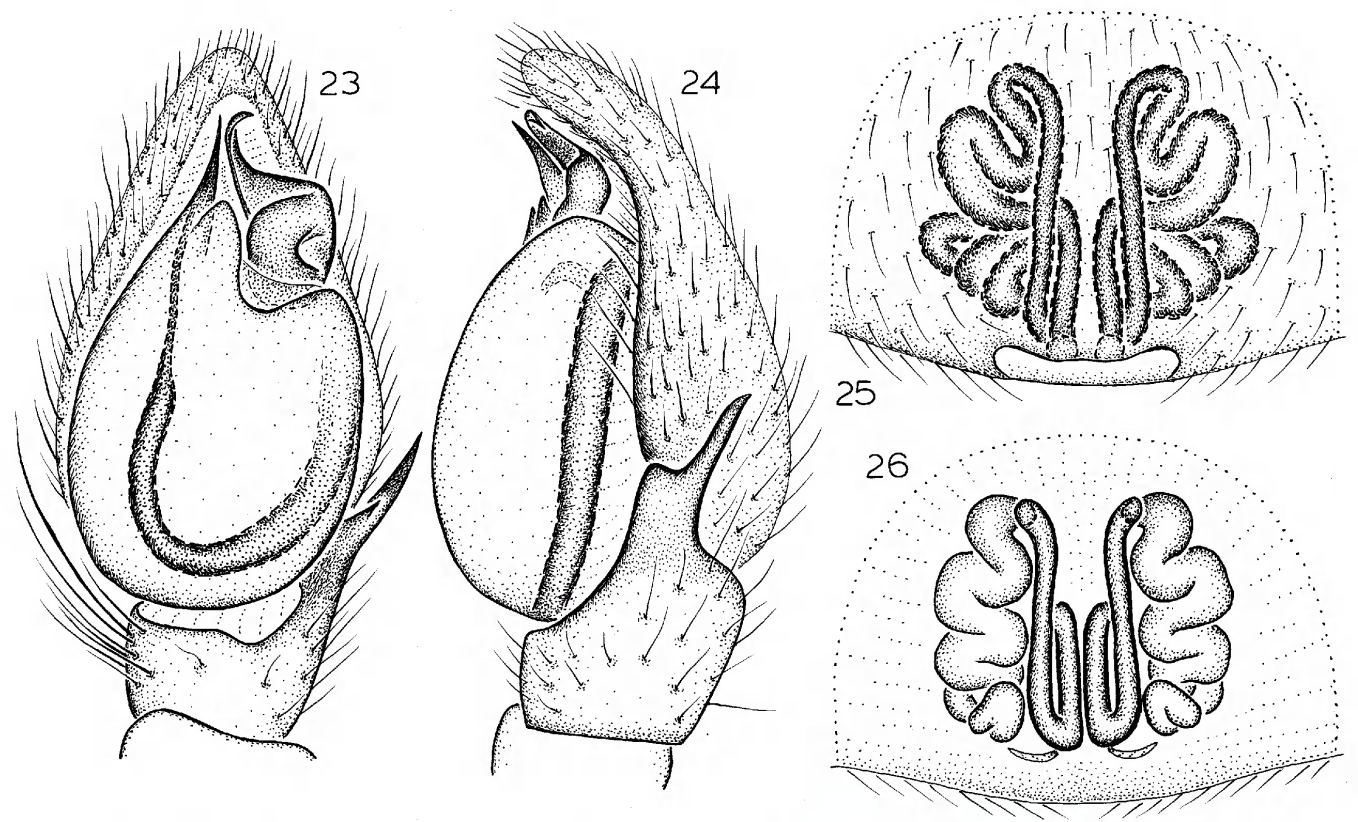

Figs. 23-26. Moreno chivato, new species. 23. Left male palp, ventral view. 24. Same, retrolateral view. 25. Epigynum, ventral view. 26. Same, dorsal view.

AMNH), 1 ; Quebrada Huaquen, Pichicuy, Oct. 16, 1992, elev. $10 \mathrm{~m}$ (N. Platnick, P. Goloboff, K. Catley, AMNH), 1 ; Zapallar, Nov. 27, 1950 (E. Ross, Michelbacher, CAS), $19 ; 4 \mathrm{~km} \mathrm{~S}$ Zapallar, $32^{\circ} 33^{\prime} \mathrm{S}$, $71^{\circ} 28^{\prime} \mathrm{W}$, July 17,1966 , coastal slope (E. Schlinger, M. Irwin, CAS), 1 ô, 1 ㅇ.

Distribution: Known only from Petorca province in Región de Valparaíso (V), Chile.

\section{Moreno grande, new species} Figures $27-30$

TyPES: Male holotype and female allotype taken at an elevation of $20 \mathrm{~m}$ on a beach 6 $\mathrm{km}$ south of Cruz Grande, Elqui, Región de Coquimbo (IV), Chile (Oct. 7, 1992; N. Platnick, P. Goloboff, K. Catley), deposited in AMNH.

ETyMology: The specific name is a noun in apposition taken from the type locality.

DIAGNOSIS: Males can be recognized by the deep but narrow notch between the dorsal and ventral prongs of the retrolateral tibial apophysis (fig. 28), females by the posteriorly produced anterior septal margin (fig. 29) and anteriorly expanded median epigynal ducts (fig. 30).

MALE: Total length 2.41. Carapace 0.94 long, 0.71 wide. Femur II 0.57 long. Coloration as in $M$. morenoi. Leg spination: femora: II p0-0-0; III p0-0-0, r0-0-0; IV p0-0-0; tibiae: II v1p-1p-2; III v0-1p-2; IV v1p-1p2; metatarsi: III, IV p0-1-1, v1p-0-0, r0-1-1. Dorsal and ventral prongs of retrolateral tibial apophysis separated by deep but narrow notch (fig. 28); median apophysis relatively wide (fig. 27).

FEMALE: Total length 2.22. Carapace 0.99 long, 0.67 wide. Femur II 0.64 long. Coloration as in $M$. morenoi. Leg spination: femora: II p0-0-0; III, IV p0-0-0, r0-0-0; tibiae: I v1p-2-2; II p0-0-1; III, IV v1p-1p-2; metatarsi: III p0-1-1, v1p-0-0, r0-1-1; IV p0-1-1, $\mathrm{v} 1 \mathrm{p}-1 \mathrm{p}-0, \mathrm{r} 0-1-1$. Anterior margin of epigynal septum produced posteriorly along midline (fig. 29); median epigynal ducts expanded anteriorly (fig. 30).

Other Material Examined: CHILE: Región de Atacama (III): Copiapó: $30 \mathrm{~km} \mathrm{~S}$ Copiapó on Route 5, Oct. 9, 1992, elev. 520 

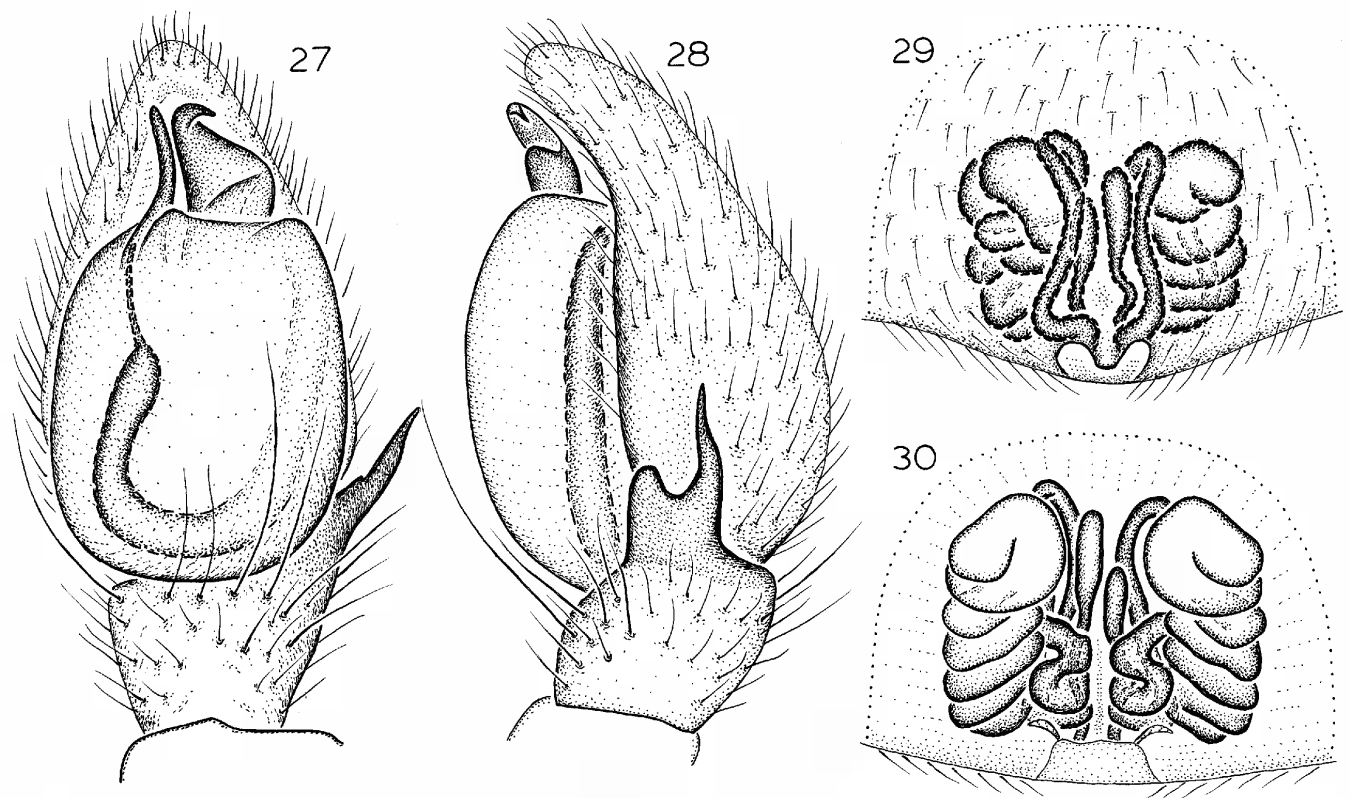

Figs. 27-30. Moreno grande, new species. 27. Left male palp, ventral view. 28. Same, retrolateral view. 29. Epigynum, ventral view. 30. Same, dorsal view.

m (N. Platnick, P. Goloboff, K. Catley, AMNH), 2o; Quebrada de León, N Caldera, Oct. 10, 1991, elev. $140 \mathrm{~m}$ (N. Platnick, P. Goloboff, K. Catley, AMNH), 10,1 .. Región de Coquimbo (IV): Elqui: beach $6 \mathrm{~km}$ S Cruz Grande, Oct. 7, 1992, elev. 20 m (N. Platnick, P. Goloboff, K. Catley, AMNH), 1ठ; El Pangue, $20 \mathrm{~km}$ S Vicuña, Oct. 4, 1992, elev. 1500 m (N. Platnick, P. Goloboff, K. Catley, AMNH), 1 ; ; La Herradura, Oct. 4, 1992, elev. $60 \mathrm{~m}$ (N. Platnick, P. Goloboff, K. Catley, AMNH), $19 ; 20$ km N La Serena on Route 5, km 491, 29 $46^{\prime} \mathrm{S}, 71^{\circ} 20^{\prime} \mathrm{W}$, Feb. 8, 1994, elev. 120 m (N. Platnick, K. Catley, R. Calderón, R. Allen, AMNH), 2 đ, 1 ㅇ․

DisTRIBUTION: Known only from northern Chile (regions III and IV).

Chileomma, new genus

TyPe SPECIES: Chileomma ruiles, new species.

ETYMOLOGY: The generic name is a contraction of Chilean Lygromma, neuter in gender.

DiAgNOSIS: The presence of only six eyes readily separates members of this genus from all other southern South American prodidomids. Elsewhere in the New World, some species of Lygromma also have only six eyes (others have eight or none), but males of that genus have a retrolateral tibial apophysis consisting of multiple, short prongs, rather than a single long prong, and females lack the anterior epigynal atrium characteristic of Chileomma.

DESCRIPTION: Small spiders, total length 1.7-2.6. Carapace oval, narrowed in front to about half its maximum width, with margins heavily sclerotized only at anterolateral corners, posterior margin slightly invaginated at pedicel, not reflexed; surface with few, short setae, without tubercles; thoracic groove short, shallow, longitudinal. Six light eyes, anterior median pair missing, anterior laterals largest, oval, posterior medians smallest, flat, irregularly rectangular, anterior laterals oval, light; from above, posterior row straight or slightly recurved, from front, posterior row slightly procurved; anterior laterals separated by slightly more than their diameter, by about 

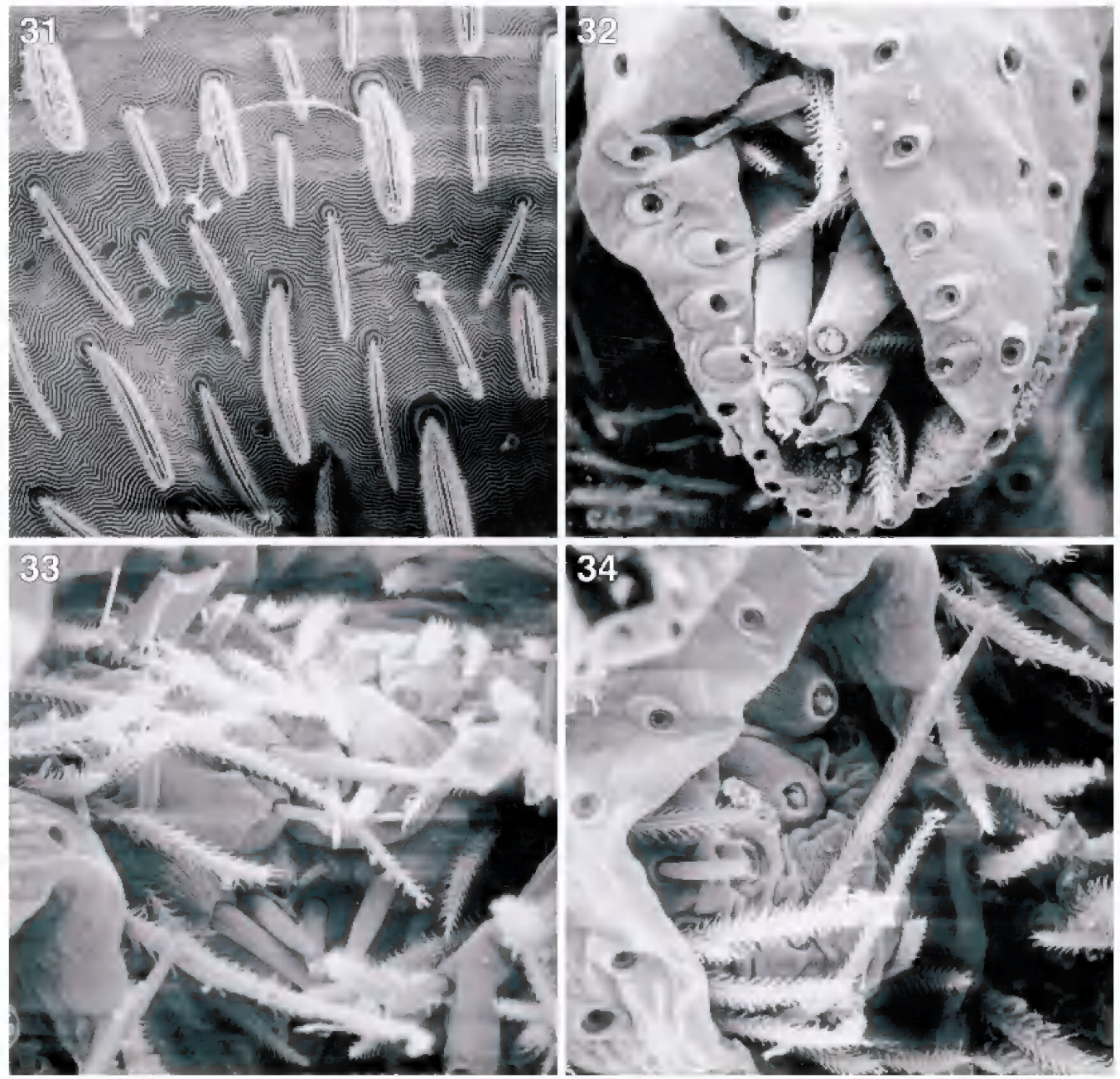

Figs. 31-34. 31. Moreno chivato, new species, female abdomen, dorsal view, showing normal and clavate setae. 32-34. Chileomma franckei, new species, female. 32. Anterior lateral spinnerets, distal view. 33. Posterior median spinnerets, distal view. 34. Posterior lateral spinnerets, distal view.

their radius from posterior medians and laterals; posterior medians separated by almost twice their maximum width, slightly closer to posterior laterals. Chelicerae vertical, not divergent, paturon with lightly sclerotized boss, promargin with row of long, curved setae, seta closest to fang base greatly elongated, distinctly bent toward midline at about one-eighth its length; promargin with three very short teeth, retromargin with row of very tiny denticles opposite fang tip, fang long, arched; chilum indistinct, apparently unipartite, triangular. Labium about as long as wide, widest posteriorly, along recurved posterior margin; anterior margin strongly rebordered. Endites moderately long, convergent anteriorly, bent and obliquely depressed near anterior margin of labium; serrula present. Sternum shield-shaped, flat, with strongly rebordered lateral margins but rebordering abruptly absent along distinct posterior protrusion between coxae IV, not expanded an- 

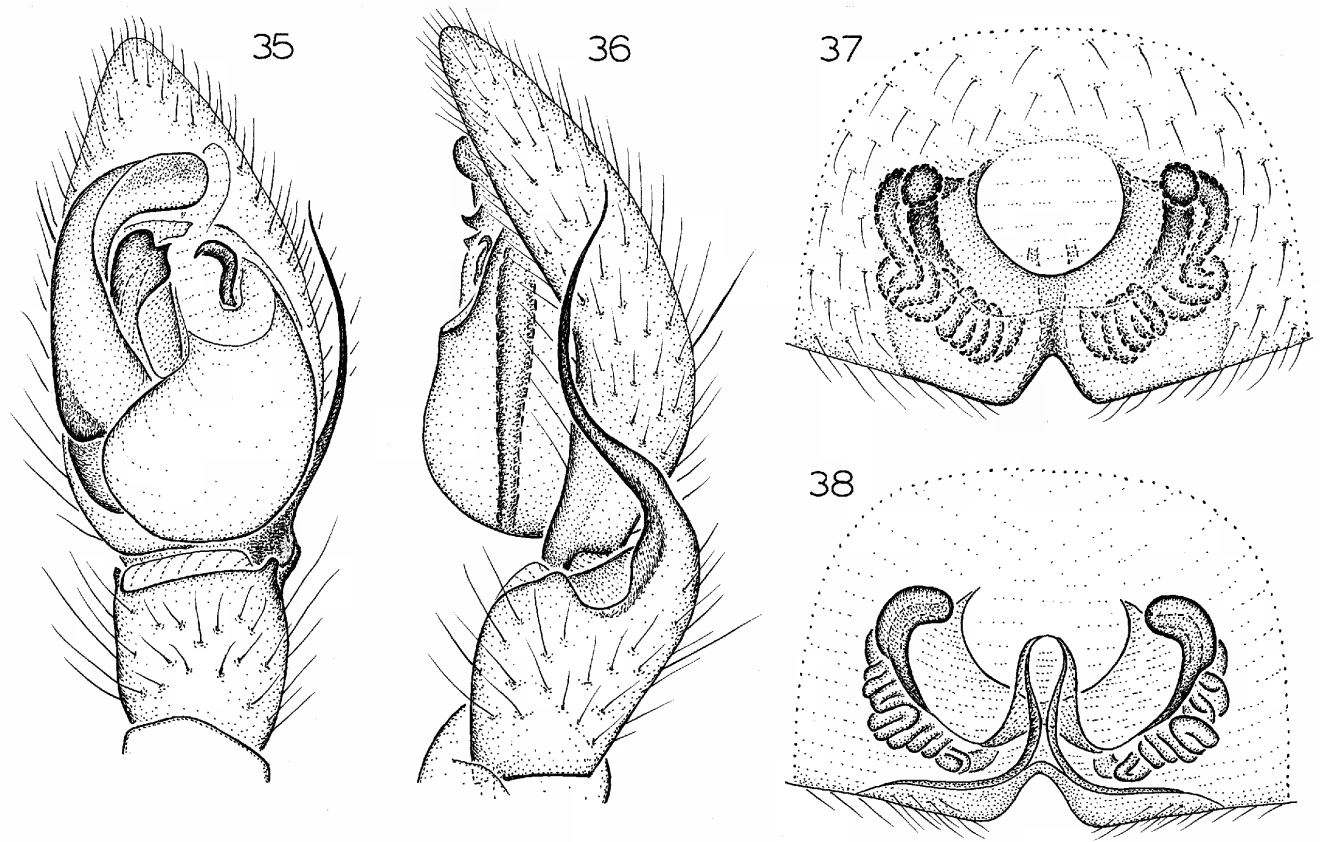

Figs. 35-38. Chileomma ruiles, new species. 35. Left male palp, ventral view. 36. Same, retrolateral view. 37. Epigynum, ventral view. 38. Same, dorsal view.

teriorly, with only indistinct extensions between coxae but with small, triangular extensions to middle of coxae; surface smooth, with long setae, posterior protrusion with numerous long, erect setae, widely separating coxae IV. Single epimeric sclerite on each side, above coxae, not extending between coxae, not fused to carapace. Pedicel composed of two dorsal sclerites (both sclerites excavated anteriorly) and sclerotized, triangular ventral sclerite almost reaching posterior tip of sternum.

Abdominal dorsum without anterior scutum in either sex, with pair of deep sigilla at about half of length; cuticle with long, recumbent setae, without clavate setae; epigastric scutum weakly sclerotized, without postepigastric sclerites, booklung covers not ridged; colulus represented by paired clusters of setae originating about halfway between anterior lateral spinnerets and wide, straight posterior spiracle. Six spinnerets, anterior laterals moderately elongated, widely separated, not advanced anteriorly, with elongate ventral tubercle arising near cuticle of basal segment, apparently bearing single major ampullate gland spigot, soft portions of cuticle bearing few piriform gland spigots with elongated bases, short shafts, and at least one accompanying seta (fig. 32); female posterior medians small, narrow, contiguous, with spigots closely clustered at tip, cluster apparently consisting of about four large cylindrical gland spigots, two minor ampullate gland spigots with long shafts, and three or four aciniform gland spigots with narrow shafts (fig. 33); female posterior laterals apparently with at least one large cylindrical gland spigot and two aciniform gland spigots (fig. 34).

Leg formula 1423, legs long, anterior pair greatly elongated, especially in males, with erect, dark setae; coxae and trochanters without dorsal tubercles, fourth trochanters only slightly elongated; anterior coxae without protuberant posterolateral corners; trochanters not notched; metatarsi and tarsi without scopula; posterior metatarsi without distal 

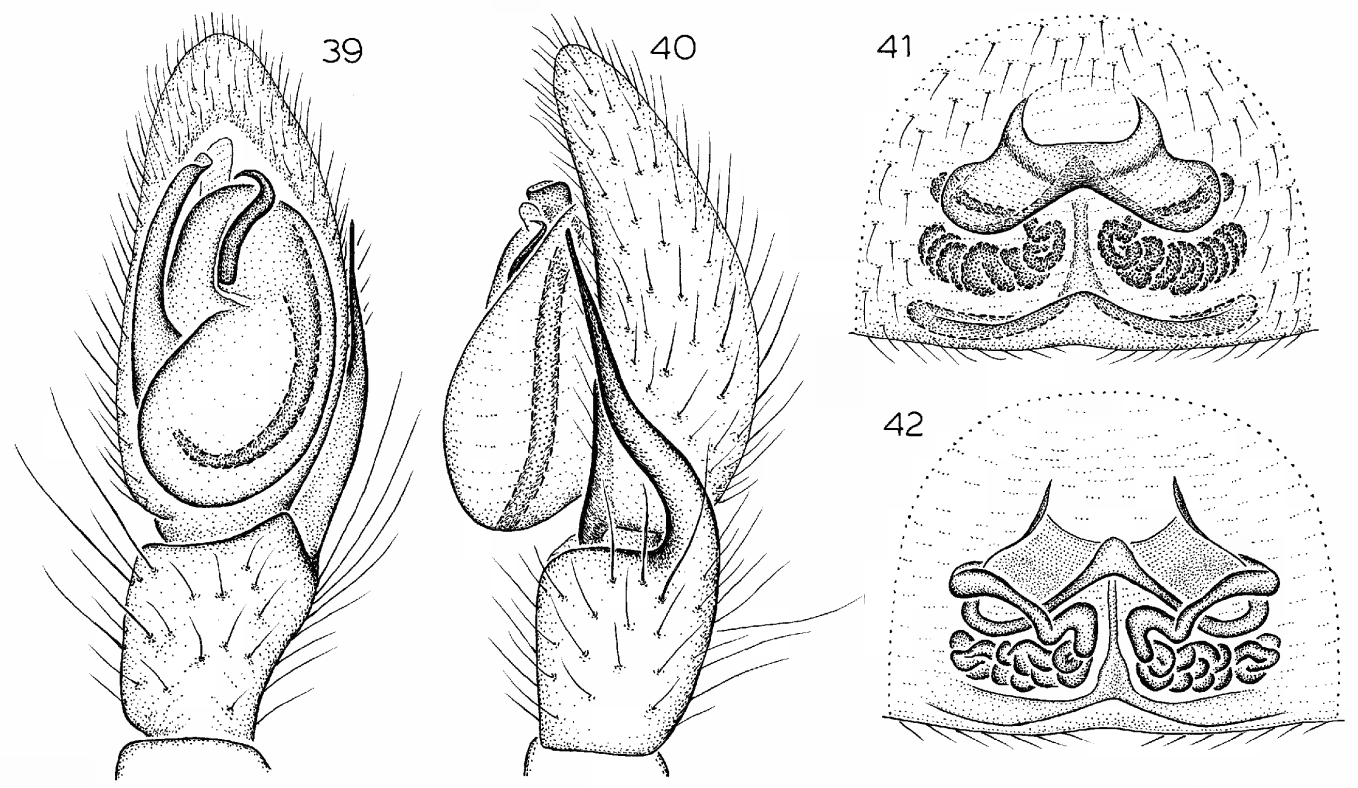

Figs. 39-42. Chileomma chilensis, new species. 39. Left male palp, ventral view. 40. Same, retrolateral view. 41. Epigynum, ventral view. 42. Same, dorsal view.

preening brushes; tarsi without cuticular cracks, with two long claws bearing two small teeth, claw tufts composed of very few, distally widened setae; dorsal surface of tarsi with unmodified proximal margin; trichobothria apparently few, in two rows dorsally on all tarsi and metatarsi. Female palpal femur, patella, tibia, and tarsus with several short spines, tarsus short, not enlarged distally, without distodorsal pad of setae, with short, apparently smooth claw. Leg spines of males not longer than those of females; typical leg spination pattern (only surfaces bearing spines listed): femora: I d1-1-0, p0-0-1; II-IV d1-1-0; tibiae: I v6-6-4; II v6-4-4; III, IV d0-1-0, p0-1-1, v2-2-2, r0-1-1; metatarsi: I, II v4-2-2; III, IV p0-1-1, v2-0-2, r0-1-1.

Male palpal tibia with long retrolateral apophysis; embolus short, originating prodistally, accompanied by transparent conductor (often difficult to observe, unless palp is at least partially expanded); median apophysis hook-shaped. Epigynum with conspicuous, anteromedian atrium; ducts highly convoluted.

\section{Chileomma ruiles, new species} Figures 35-38

TYPES: Male holotype and female allotype taken at an elevation of $160 \mathrm{~m}$ in the Reserva Nacional Los Ruiles, Cauquenes, Región del Maule (VII), Chile (Feb. 25, 1992; N. Platnick, P. Goloboff, M. Ramírez), deposited in AMNH.

ETYMOLOGY: The specific name is a noun in apposition taken from the type locality.

DIAGNOSIS: Males can easily be recognized by the whip-shaped tip of the retrolateral tibial apophysis (fig. 36), females by the rounded epigynal atrium (fig. 37) and laterally situated ducts (fig. 38).

MALE: Total length 2.18. Carapace 1.06 long, 0.72 wide. Femur II 0.84 long. Carapace and mouthparts light yellow except for black patch uniting triad of eyes of each side, sternum paler, abdomen white, legs light yellow. Leg spination: tibia IV d1-1-0, v1p-2-2. Retrolateral tibial apophysis with whipshaped tip (fig. 36); embolus relatively wide (fig. 35).

Female: Total length 2.19. Carapace 1.08 

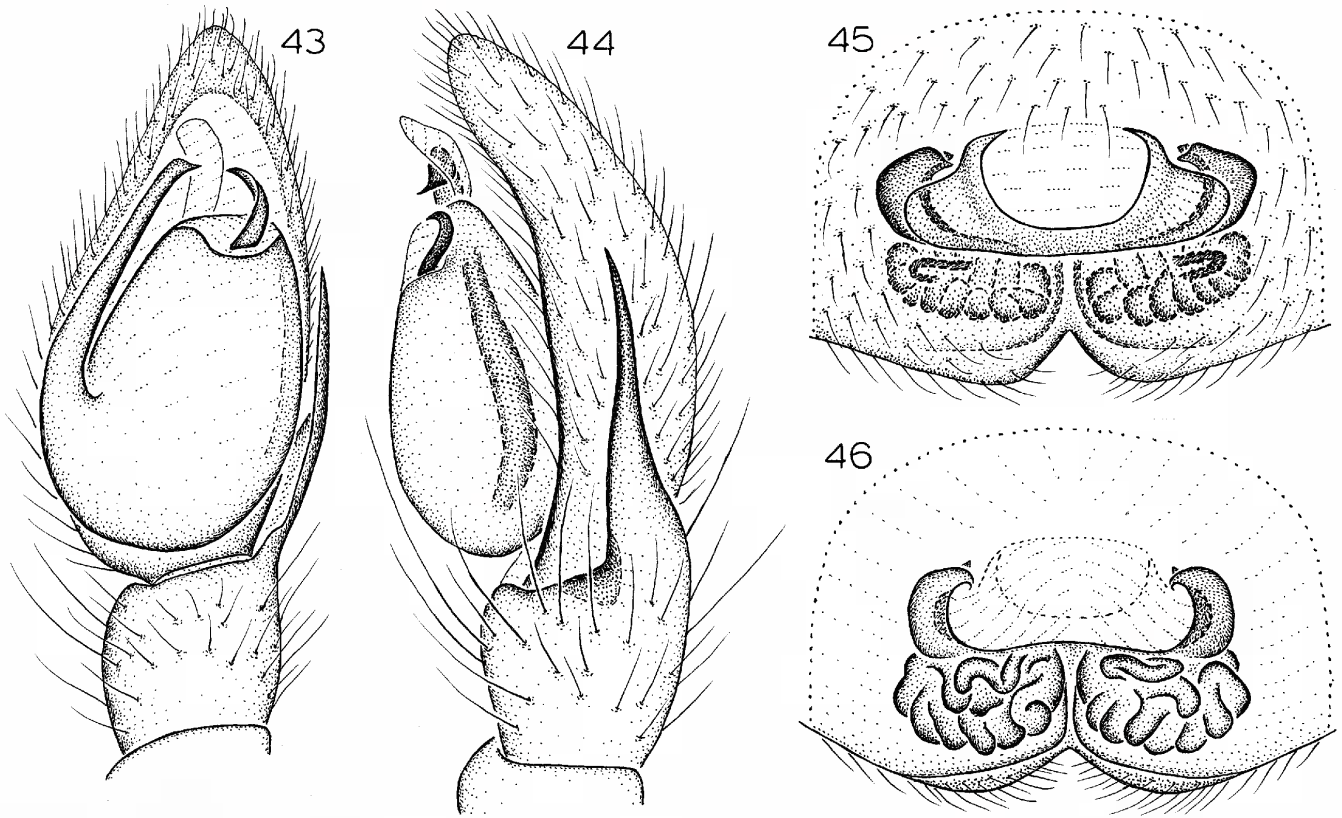

Figs. 43-46. Chileomma malleco, new species. 43. Left male palp, ventral view. 44. Same, retrolateral view. 45. Epigynum, ventral view. 46. Epigynum, dorsal view.

long, 0.79 wide. Femur II 0.82 long. Coloration as in male. Leg spination: tibiae: I v66-2; II v4-4-4; III v1p-2-2; IV d1-1-0, v1p2-2; metatarsi: II v2-2-2. Epigynal atrium rounded (fig. 37); ducts situated laterally (fig. 38).

Other MATERIAL ExAmined: Two males taken with the types (AMNH).

Distribution: Known only from the type locality in central Chile.

\section{Chileomma chilensis, new species} Figures 39-42

TYPE: Male holotype taken in a xerophytic forest at an elevation of $200 \mathrm{~m} 8 \mathrm{~km} \mathrm{~W}$ Florida, $36^{\circ} 49^{\prime} \mathrm{S}, 72^{\circ} 44^{\prime} \mathrm{W}$, Concepción, Región del Bío-Bío (VIII), Chile (Jan. 18, 1995; N. Platnick, K. Catley, D. Silva), deposited in AMNH.

ETYMOLOGY: The specific name refers to the type locality.

DiAGNOSIS: Males can easily be recognized by the relatively narrow embolus (fig. 39), females by the short, wide epigynal atrium (fig. 41) and anterolaterally elongated ducts (fig. 42).

MALE: Total length 2.38. Carapace 0.98 long, 0.66 wide. Femur II 0.73 long. Coloration as in $C$. ruiles. Leg spination: tibiae: I v6-4-4; II v4-4-2; III, IV v1p-2-2; metatarsi: III r0-1-0. Retrolateral tibial apophysis reaching almost to tip of cymbium, relatively narrow at base (fig. 40); embolus relatively narrow (fig. 39).

FEMAlE: Total length 2.53. Carapace 1.06 long, 0.87 wide. Femur II 0.71 long. Coloration as in $C$. ruiles. Leg spination: tibiae: I v6-4-4; II v4-4-2; III v1p-2-2; IV d1-1-0, v1p-2-2; metatarsi III, IV r0-1-0. Epigynal atrium relatively short (fig. 41); ducts anterolaterally elongated (fig. 42).

Other Material ExAmined: CHILE: Región del Bío-Bío (VIII): Concepción: 8.4 km W La Florida, Jan. 2, 1983, Berlese, leaf and $\log$ litter, subtropical xerophytic forest, elev. $170 \mathrm{~m}$ (A. Newton, M. Thayer, AMNH), 1 오.

Distribution: Known only from Concepción, Chile. 

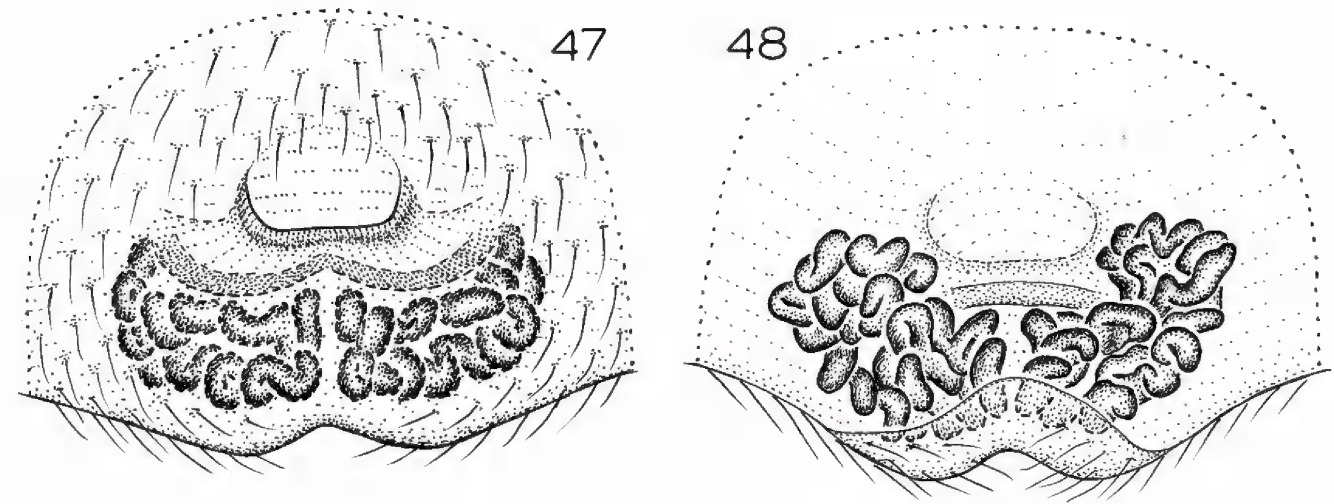

Figs. 47, 48. Chileomma franckei, new species. 47. Epigynum, ventral view. 48. Epigynum, dorsal view.

Chileomma malleco, new species Figures 43-46

TYPE: Female holotype taken at an elevation of $740 \mathrm{~m}$ at Alto Caledonia, $42 \mathrm{~km} \mathrm{E}$ Mulchen, Malleco, Región de La Araucanía (IX), Chile (Feb. 14, 1992; N. Platnick, P. Goloboff, M. Ramírez), deposited in AMNH.

ETYMOLOGY: The specific name is a noun in apposition taken from the type locality.

DiAGNOSIS: Males resemble those of $C$. chilensis but have a proximally wider retrolateral tibial apophysis (fig. 44) and a shorter median apophysis (fig. 43); females can be recognized by the wide epigynal atrium (fig. 45) and posteriorly situated ducts (fig. 46).

Male: Total length 2.02. Carapace 0.95 long, 0.77 wide. Femur II 0.69 long. Coloration as in $C$. ruiles. Leg spination: tibiae: I v4-4-4; II v4-4-2; III v1p-2-2; IV d1-1-0, v1p-2-2; metatarsi: I, II v2-2-2. Retrolateral tibial apophysis reaching almost to tip of cymbium, relatively wide at base (fig. 44); median apophysis relatively short (fig. 43).

Female: Total length 2.52. Carapace 1.15 long, 0.81 wide. Femur II 0.86 long. Coloration as in $C$. ruiles. Leg spination: tibiae: I, II v6-4-2; III v1p-2-2; IV d1-1-0, v1p-2-2; metatarsi: II v2-2-2; IV r0-1-0. Epigynal atrium very wide (fig. 45); ducts restricted to posterior half of epigynum (fig. 46).

Other Material Examined: CHILE: Región de La Araucanía (IX): Malleco: Monumento Nacional Contulmo, 38 $00^{\prime} 47^{\prime \prime} \mathrm{S}$, $73^{\circ} 11^{\prime} 15^{\prime \prime} \mathrm{W}$, Dec. 19-21, 1998 (M. Ramírez,
L. Compagnucci, G. Grismado, L. Lopardo, MACN 10596), 10 , Feb. 10-11, 2005, Berlese, elev. 360 m (M. Ramírez, F. Labarque, MACN ARAMR000193), 1 오.

Distribution: Known only from Malleco, Chile.

Chileomma campana, new species Figures 49-52

TYPE: Female holotype taken in a hygrophyll forest at an elevation of $1000 \mathrm{~m}$ at Cerro de La Campana, San Felipe de Aconcagua, Región de Valparaíso (V), Chile (Dec. 11, 1979; G. Betancourt), deposited in AMNH.

Eтymology: The specific name is a noun in apposition taken from the type locality.

DIAGNOSIS: Males can easily be recognized by the prolaterally extended embolus tip (fig. 49), females by the short epigynal atrium and anteriorly expanded lateral epigynal ducts (figs. 51, 52).

MALE: Total length 2.27. Carapace 0.97 long, 0.77 wide. Femur II 0.70 long. Coloration as in $C$. ruiles. Leg spination (leg I missing): tibiae: II v6-4-4; III v1p-2-2, r0-10; IV v1p-2-2; metatarsi: III p0-0-1; IV r01-0. Retrolateral tibial apophysis angular, reaching to about half of cymbial length (fig. 50 ); embolar tip produced prolaterally (fig. 49).

Female: Total length 2.42. Carapace 0.95 long, 0.72 wide. Femur II 0.71 long. Coloration as in $C$. ruiles. Leg spination (leg IV 

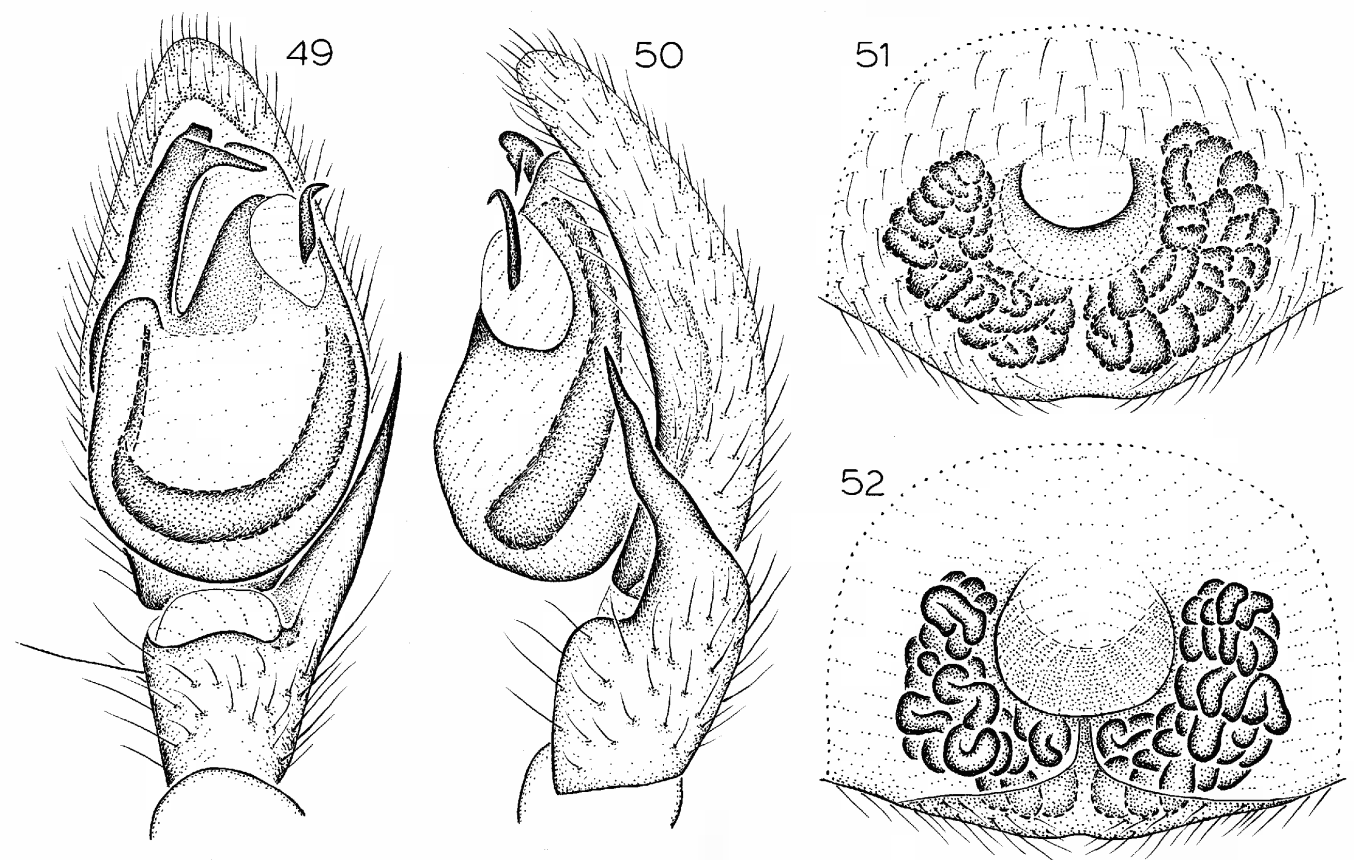

Figs. 49-52. Chileomma campana, new species. 49. Left male palp, ventral view. 50. Same, retrolateral view. 51. Epigynum, ventral view. 52. Same, dorsal view.

missing): tibiae: I v6-4-4; III v1p-2-2, r0-10 . Epigynal atrium relatively short, wide (fig. 51), lateral ducts extending to anterior edge of atrium (fig. 52).

Other Material Examined: CHILE: Región de Valparaíso (V): San Felipe de Aconcagua: Cerro de La Campana, Mar. 12, 1979, sclerophyll forest, elev. 1060 m (G. Betancourt, AMNH), 30 .

DistRIBUTION: Known only from Aconcagua, Chile.

\section{Chileomma franckei, new species} Figures 32-34, 47, 48

TYPE: Female holotype from a Berlese sample of leaf litter taken at an elevation of $8 \mathrm{~m}$ in a small, mixed forest $11 \mathrm{~km} \mathrm{E}$ of Quintero, Valparaíso, Región de Valparaíso (V), Chile (Jan. 10, 1985; N. Platnick, O. Francke), deposited in AMNH.

ETYMOLOGY: The specific name is a patronym in honor of one of the collectors of the holotype, Dr. Oscar Francke.
DiAGNOSIS: Males are unknown; females resemble those of $C$. chilensis in having a relatively short, wide epigynal atrium (fig. 47 ), but have anteriorly more convoluted epigynal ducts (fig. 48).

MALE: Unknown.

Female: Total length 1.83. Carapace 0.74 long, 0.59 wide. Femur II 0.53 long. Coloration as in C. ruiles. Leg spination: tibiae: I v6-4-4; II v4-4-2; III r0-1-0; IV v1p-2-2; metatarsi: III, IV p1-0-1, r1-0-1. Epigynal atrium short, wide (fig. 47), lateral ducts convoluted throughout their length (fig. 48).

Other Material Examined: CHILE: Región de Valparaíso (V): Quillota: Cuesta

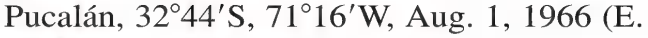
Schlinger, M. Irwin, CAS), 2 \% .

DisTRIBUTION: Known only from Quillota and Valparaíso, Chile.

\section{Chileomma petorca, new species} Figures 53, 54

TYPE: Female holotype taken in pitfall trap at an elevation of $10 \mathrm{~m}$ at Quebrada Hua- 

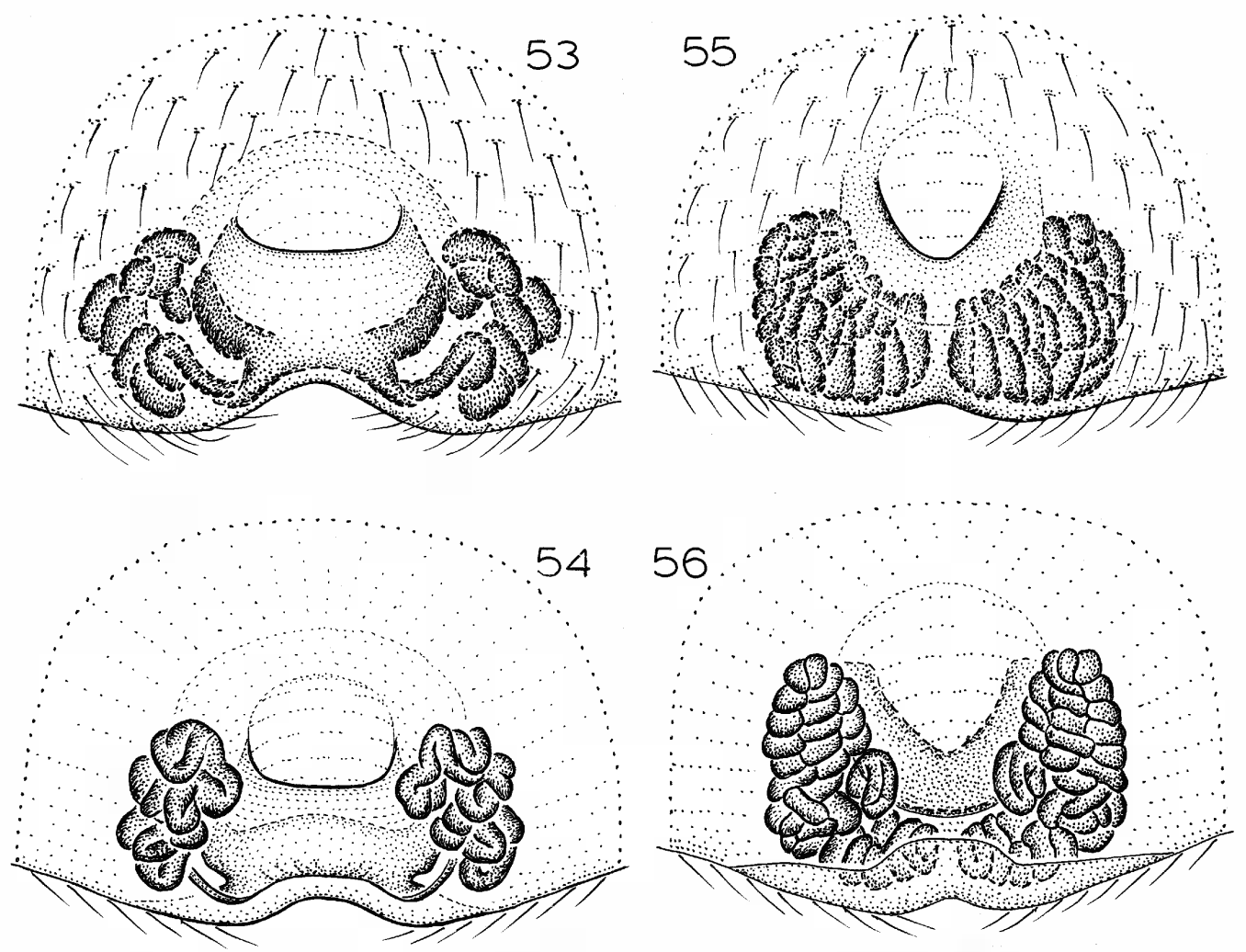

Figs. 53-56. 53, 54. Chileomma petorca, new species, female epigynum. 55, 56. Chileomma rinconada, new species, same. 53, 55. Ventral view. 54, 56. Dorsal view.

quen, Pichicuy, Petorca, Región de Valparaíso (V), Chile (Oct. 2-16, 1992; N. Platnick, P. Goloboff, K. Catley), deposited in AMNH.

ETYMOLOGY: The specific name is a noun in apposition taken from the type locality.

DIAGNOSIS: Males are unknown; females can be recognized by the relatively large and only loosely convoluted lateral epigynal ducts (fig. 54).

MALE: Unknown.

Female: Total length 1.76. Carapace 0.75 long, 0.66 wide. Femur II 0.62 long. Coloration as in $C$. ruiles. Leg spination: tibiae: II v6-4-2; III v1p-2-2; IV d1-1-0, v1p-2-2. Epigynal atrium short, wide (fig. 53); lateral ducts loosely convoluted, extending to about half of atrial length (fig. 54).

Other Material Examined: One female taken with the holotype (AMNH).
Distribution: Known only from Petorca, Chile.

\section{Chileomma rinconada, new species} Figures 55, 56

TYPE: Female holotype from Quebrada de La Plata, La Rinconada, near Maipu, $33^{\circ} 30^{\prime} \mathrm{S}, 70^{\circ} 55^{\prime} \mathrm{W}$, Santiago, Región Metropolitana de Santiago, Chile (Sept. 28, 1966; E. Schlinger), deposited in CAS.

ETYMOLOGY: The specific name is a noun in apposition taken from the type locality.

DiAGNOSIS: Males are unknown; females can be recognized by the diamond-shaped epigynal atrium (fig. 55) and wide, triangular masses of lateral epigynal ducts (fig. 56).

MALE: Unknown.

Female: Total length 2.42. Carapace 1.12 
long, 0.86 wide. Femur II 0.82 long. Coloration as in $C$. ruiles. Leg spination: tibiae: I v6-4-4; III v1p-2-2; metatarsi I v4-4-2. Epigynal atrium diamond-shaped (fig. 55); lateral ducts forming triangular masses (fig. 56).

Other MATERIAl Examined: None.

DistRIBUTION: Known only from Santiago, Chile.

\section{Chilongius, new genus}

TyPe SPECIES: Chilongius palmas, new species.

ETYMOLOGY: The generic name is a contraction of Chilean Tricongius, male in gender.

DiAGNOSIS: The combination of eight eyes, dentate tarsal claws, and an abdomen without translucent clavate setae separates members of this genus from the other Chilean theumines. Males can be recognized by the short, wide, curved, prodistally situated embolus (as in figs. 63, 67), females by the small, centrally situated epigynal atrium (as in figs. 65, 69).

DesCrIPTION: Medium-sized spiders, total length 3.3-5.6. Carapace oval, narrowed in front to only about two-thirds its maximum width, with margins heavily sclerotized only at anterolateral corners, posterior margin slightly invaginated at pedicel, slightly reflexed; pars thoracica coated with long, recumbent setae, without tubercles; thoracic groove long, shallow, longitudinal. Eight eyes in two rows, anterior medians smallest, circular, dark, about half the size of other light, subequal, eyes; lateral pairs oval, posterior medians flat, irregularly rectangular; from above, both eye rows slightly recurved, from front, both rows slightly procurved; anterior medians separated by slightly more than their diameter, about as far from anterior laterals; posterior medians separated by about twice their width, about as far from posterior laterals; anterior and posterior laterals separated by their width; median ocular quadrangle much wider in back than in front, much wider in back than long. Chelicerae slightly projecting, slightly divergent, paturon with distinct, lightly sclerotized boss, promargin with row of long, curved setae, seta closest to fang base greatly elongated, distinctly bent toward midline at about oneeighth its length, many others on slight widening opposite base of fang; promargin with three strong teeth, distal tooth largest, retromargin with two small, subequal teeth, distal tooth situated distad of most distal promarginal tooth; fang long, arched; chilum short, wide, unipartite. Labium slightly wider than long, widest posteriorly, along straight posterior margin; anterior margin strongly rebordered. Endites moderately long, convergent anteriorly, bent and obliquely depressed near anterior margin of labium; serrula present. Sternum shield-shaped, flat, with strongly rebordered lateral margins but rebordering abruptly absent along distinct posterior protrusion between coxae IV, not expanded anteriorly, with distinct extensions to and between coxae; surface smooth, with short, erect setae, lateral margins and posterior protrusion with numerous long, erect setae, coxae IV widely separated. Single epimeric sclerite on each side, above coxae, slightly extended between coxae, not fused to carapace. Pedicel composed of two dorsal sclerites (both sclerites excavated anteriorly) and sclerotized, triangular ventral sclerite almost fused to posterior tip of sternum.

Abdominal dorsum without anterior scutum in either sex, with two pairs of sigilla in anterior one-third of length; cuticle with long, recumbent setae, without clavate setae; epigastric scutum weakly sclerotized, with tiny, triangular, seta-bearing postepigastric sclerites, booklung covers not ridged; colulus absent, posterior spiracle situated well anterior of spinnerets, not as wide as separation between anterior lateral spinnerets. Six spinnerets, anterior laterals moderately elongated, widely separated, not advanced anteriorly, with elongate ventral tubercle arising near cuticle of basal segment, bearing large major ampullate gland spigot closely accompanied by second smaller, possibly nonfunctional, major ampullate gland spigot, soft portions of cuticle bearing about eight piriform gland spigots with elongated bases, short shafts, and at least two accompanying setae (fig. 57); female posterior medians small, narrow, contiguous, with spigots closely clustered at tip, cluster apparently consisting of at least one large cylindrical gland spigot, one minor ampullate gland spigot with short shaft, and 

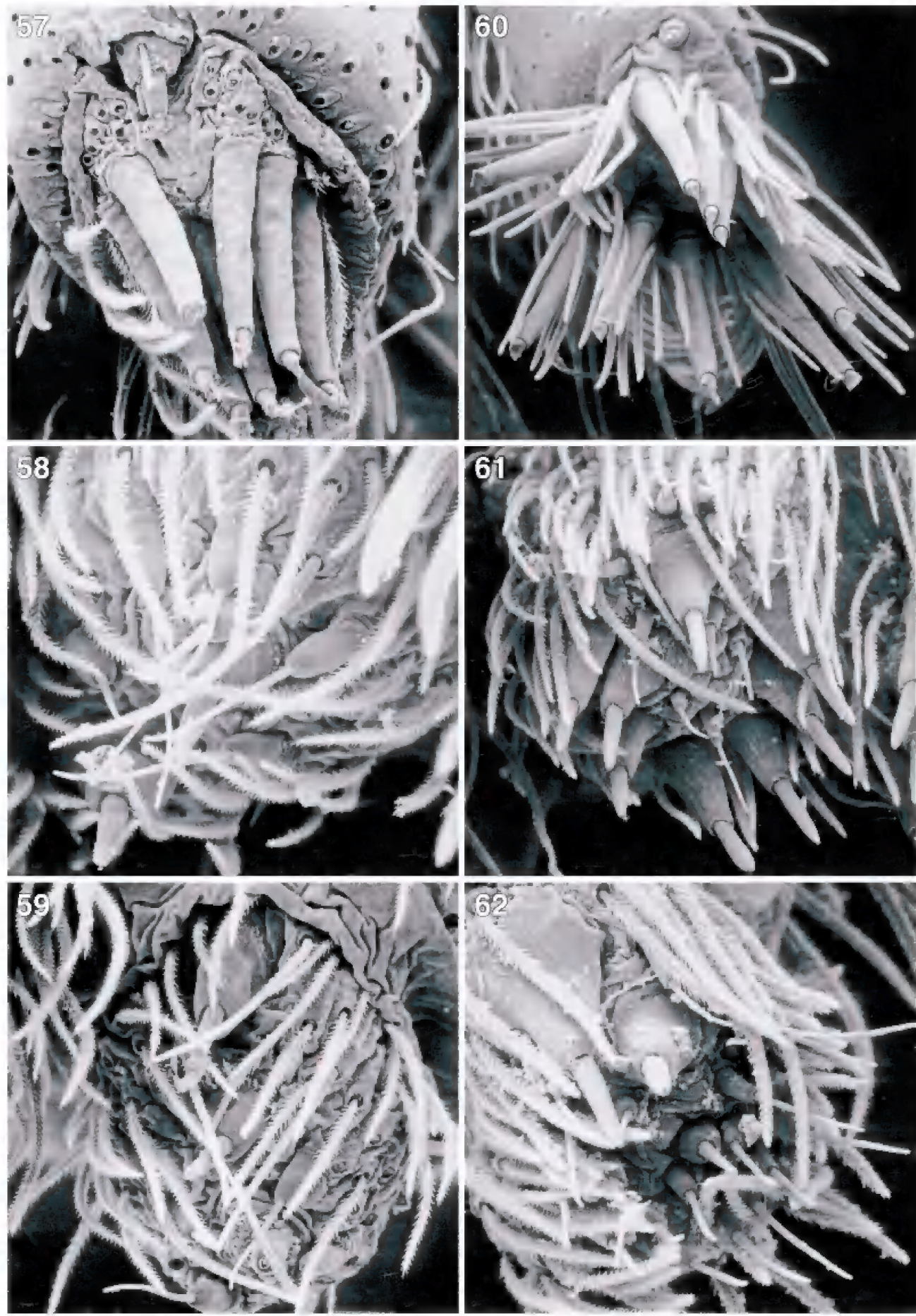
around six aciniform gland spigots with long, narrow shafts (fig. 58); female posterior laterals apparently with two large cylindrical gland spigots and around four more distally situated aciniform gland spigots with narrower shafts (fig. 59).

Leg formula 4123, legs long, anterior pair greatly elongated in males, with erect, dark setae; coxae and trochanters without dorsal tubercles, fourth trochanters only slightly elongated; anterior coxae without protuberant posterolateral corners; trochanters not notched; anterior metatarsi and tarsi with distinct ventral scopula; posterior metatarsi without distal preening brushes; tarsi without cuticular cracks, with two long claws bearing normal teeth and more proximal set of closely spaced, proximally directed teeth, most proximal of which clasps base of modified seta (figs. 7, 8), claw tufts each composed of about six distally widened setae; dorsal surface of tarsi with rebordered proximal margin; trichobothria apparently few, long, in two rows dorsally on all tarsi and metatarsi. Female palpal femur, patella, tibia, and tarsus with several weak spines, tarsus long, not enlarged distally, without distodorsal pad of setae, with long claw bearing few small teeth along middle third of its length. Leg spines of males not longer than those of females; typical leg spination pattern (only surfaces bearing spines listed): femora: I d1-1-0, p00-1; II d1-1-0, p0-1-2; III d1-1-1, p0-1-1, r00-1; IV d1-1-1, p0-1-1, r0-1-1; tibiae: II v1p1p-0; III, IV d0-1-0, p0-1-1, v2-2-2, r1-1-1; metatarsi: II v2-0-0; III p1-1-2, v2-2-2, r0-11; IV p1-2-2, v2-2-2, r1-2-1.

Male palpal tibia with long retrolateral apophysis; embolus wide, curved, originating prodistally, accompanied by transparent conductor; median apophysis relatively long, distally hooked. Epigynum with conspicuous but small, medially situated atrium; ducts highly convoluted but confined to posterior half of epigynum.
Chilongius palmas, new species

Figures 7, 8, 57-59, 63-66

TyPEs: Male holotype and female allotype taken in pitfall trap at unburned site at Palmas de Ocoa, Parque Nacional La Campana, San Felipe de Aconcagua, Región de Valparaíso, Chile (Dec. 21, 1984; R. Calderón), deposited in AMNH.

ETYMOLOGY: The specific name is a noun in apposition taken from the type locality.

DiAGNOSIS: Males can be recognized by the relatively long, narrow, gradually tapering embolus (fig. 63), females by the thumbshaped median epigynal ducts (fig. 66).

MALE: Total length 4.49. Carapace 2.23 long, 1.70 wide. Femur II 1.62 long. Carapace and mouthparts light brownish orange, sternum paler, abdomen beige, legs light yellow. Leg spination: femora: II p0-0-2; III r01-1; IV r0-0-1; tibiae: II v1r-1r-2; III r0-1-1; metatarsi: III p0-2-2. Retrolateral tibial apophysis distally narrowed (fig. 64); embolus relatively long, narrow, gradually narrowing toward tip (fig. 63).

FEMALE: Total length 5.61. Carapace 2.16 long, 1.70 wide. Femur II 1.54 long. Coloration as in male. Leg spination: II p0-0-2; III d1-1-0, r0-1-1; IV r0-0-1; tibiae: v1r-2-0; III r0-1-1. Epigynal atrium relatively narrow, triangular (fig. 65); paramedian epigynal ducts thumb-shaped, all ducts situated well posterior of posterior atrial margin (fig. 66).

OTHER MATERIAL EXAMINED: CHILE: Región de Valparaíso (V): San Felipe de Aconcagua: Palmas de Ocoa, Parque Nacional La Campana, Dec. 21, 1984, Jan. 29, 1985, Mar. 14, 1985, Apr. 12, 1985, July 19, 1985, Aug. 23, 1985, pitfalls, unburned site (R. Calderón, AMNH), 6 \% , Oct. 26-Dec. 21, 1984, pitfalls, burned and unburned sites (R. Calderón, AMNH), 20 đ. Valparaíso: $11 \mathrm{~km}$ E Quintero, Jan. 10, 1985, small mixed forest, elev. $8 \mathrm{~m}$ (N. Platnick, O. Francke, AMNH), 1 ; Río Marga Marga, Los Perales, $33^{\circ} 09^{\prime} \mathrm{S}, 71^{\circ} 19^{\prime} \mathrm{W}$, Oct. 13 , 1966, elev. 330

Figs. 57-62. 57-59. Chilongius palmas, new species, female. 60-62. Chileuma renca, new species, female. 57, 60. Anterior lateral spinnerets, distal view. 58, 61. Posterior median spinnerets, distal view. 59, 62. Posterior lateral spinnerets, distal view. 

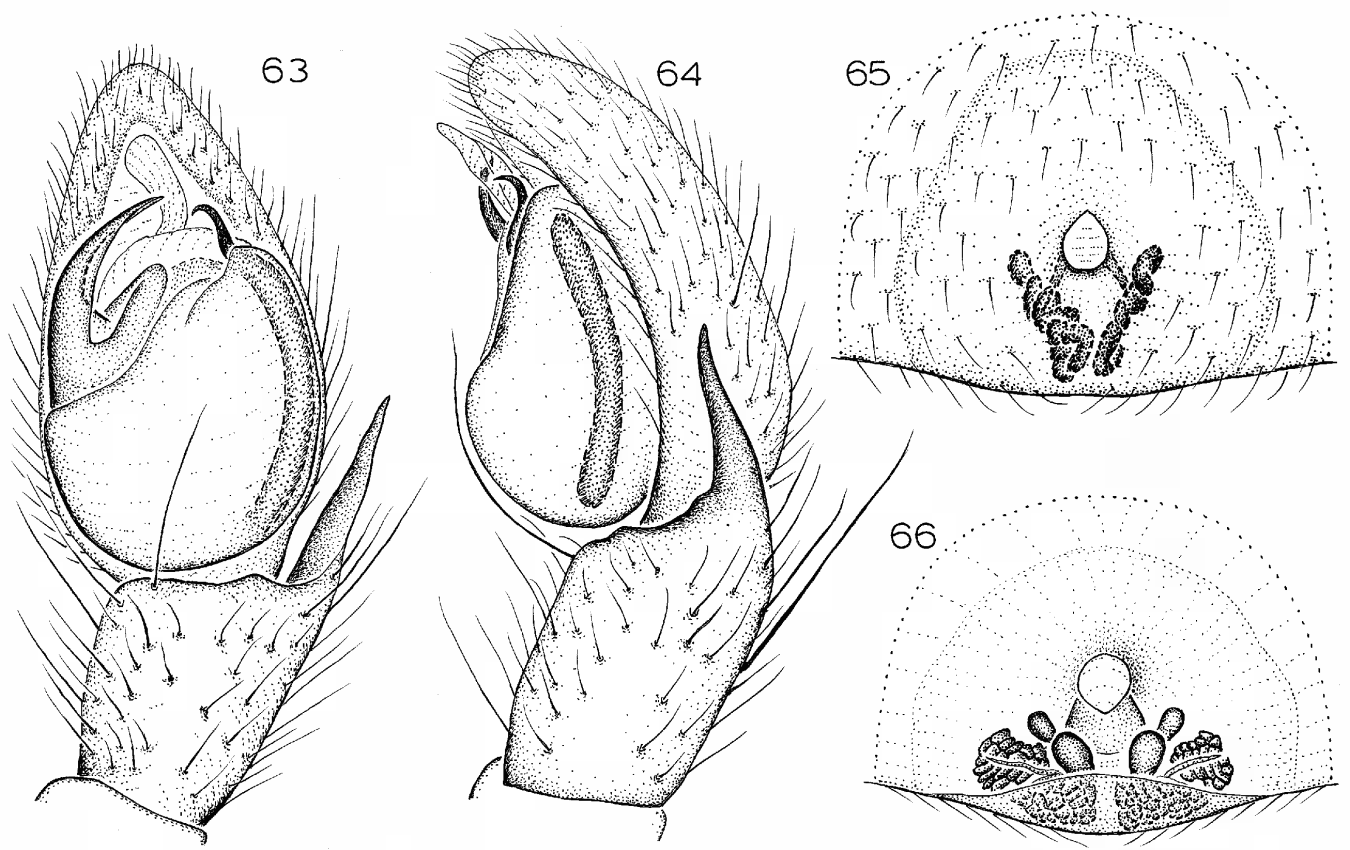

Figs. 63-66. Chilongius palmas, new species. 63. Left male palp, ventral view. 64. Same, retrolateral view. 65. Epigynum, ventral view. 66. Same, dorsal view.

m (M. Irwin, E. Schlinger, CAS), $10^{\star}$; Ventana, May 13, 1961 (R. Donoso, A. Archer, AMNH), 1 ㅇ․

Distribution: Known only from Aconcagua and Valaparaíso, Chile.

\section{Chilongius molles, new species Figures 67-70}

TyPES: Male holotype and female allotype taken at an elevation of $10 \mathrm{~m}$ at Los Molles, Route $5, \mathrm{~km} 188,32^{\circ} 14^{\prime} \mathrm{S}, 71^{\circ} 30^{\prime} \mathrm{W}$, Petorca, Región de Valparaíso (V), Chile (Nov. 9, 1993; N. Platnick, K. Catley, M. Ramírez, R. Allen), deposited in AMNH.

ETYMOLOGY: The specific name is a noun in apposition taken from the type locality.

DIAGNOSIS: Males can be distinguished by the distally wide and curved embolus (fig. 67), females by the relatively wide, posteriorly rebordered epigynal atrium (fig. 69) and wide internal epigynal septum (fig. 70).

MALE: Total length 3.45. Carapace 1.61 long, 1.28 wide. Femur II 1.11 long. Coloration as in C. palmas. Leg spination: femora: II d1-0-0, p0-0-1; IV r0-0-1; tibiae: II v0-2-
0; III r0-1-1; IV p0-1-1; metatarsi: I v2-0-0; III p0-1-2, v1p-2-2; IV p0-1-2, v1p-2-2, r1$1-2$. Retrolateral tibial apophysis distally narrowed (fig. 68); embolus relatively long, wide throughout its length (fig. 67).

FEMALE: Total length 4.50. Carapace 2.00 long, 1.54 wide. Femur II 1.34 long. Coloration as in C. palmas. Leg spination: femora: II p0-0-1; III d1-1-0; IV d1-1-0, r0-0-1; tibiae: II v0-0-0; III p1-1-1, r0-1-1; metatarsi: II v0-0-0; III p0-1-2, r0-1-2; IV p0-2-2, r0$2-1$. Epigynal atrium rectangular, with rebordered posterior margin (fig. 69); paramedian epigynal ducts thumb-shaped, separated by wide septum (fig. 70).

Other Material Examined: CHILE: Región de Coquimbo (IV): Choapa: Ñague, $10 \mathrm{~km} \mathrm{~N}$ Los Vilos, Route 5, km 236, $31^{\circ} 50^{\prime} \mathrm{S}, 71^{\circ} 31^{\prime} \mathrm{W}$, Nov. 13, 1993, elev. $40 \mathrm{~m}$ (N. Platnick, K. Catley, M. Ramírez, R. Allen, AMNH), 2․ Región de Valparaíso (V): Petorca: Los Molles, Route 5, km 188, $32^{\circ} 14^{\prime} \mathrm{S}, 71^{\circ} 30^{\prime} \mathrm{W}$, Nov. 9, 1993, elev. $10 \mathrm{~m}$ (N. Platnick, K. Catley, M. Ramírez, R. Allen, AMNH), 29, Jan. 27, 1994, elev. 10 m 

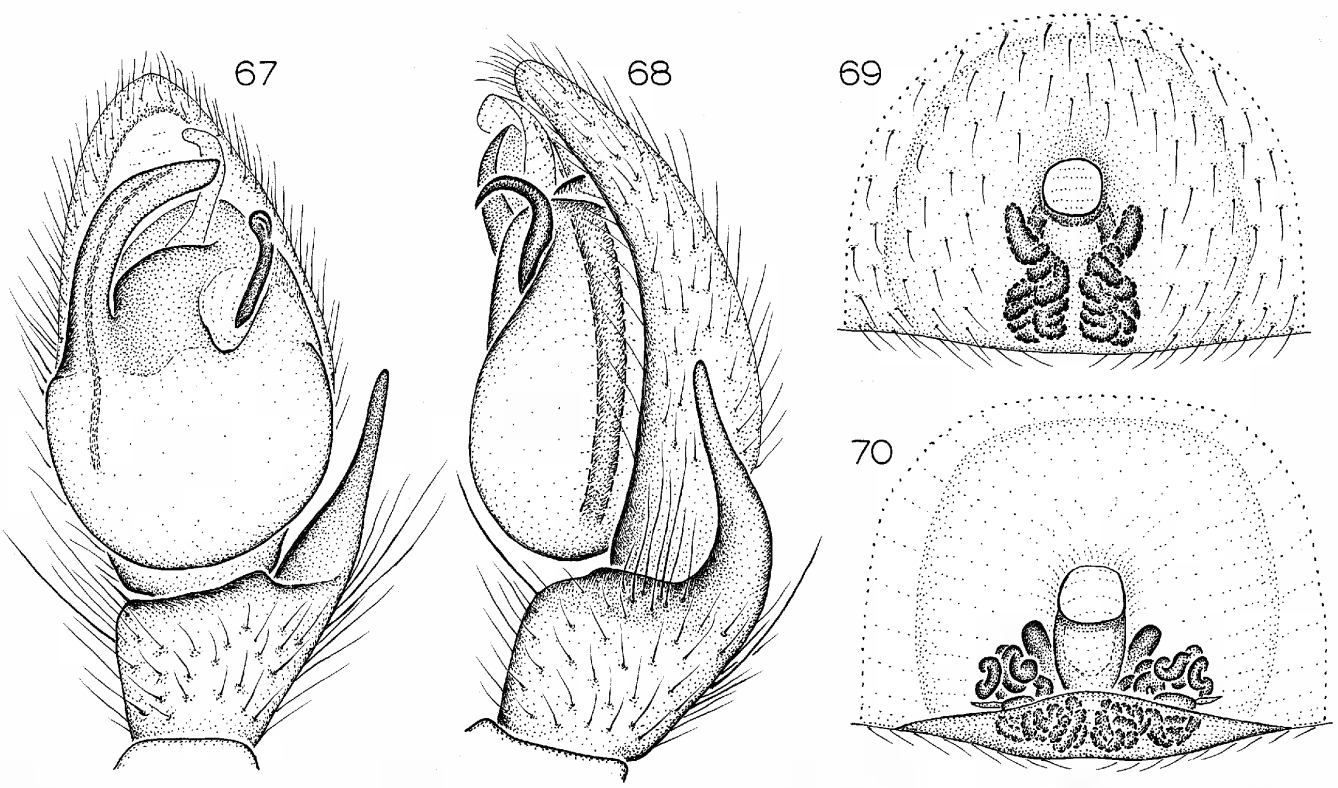

Figs. 67-70. Chilongius molles, new species. 67. Left male palp, ventral view. 68. Same, retrolateral view. 69. Epigynum, ventral view. 70. Same, dorsal view.

(N. Platnick, K. Catley, R. Calderón, R. Allen, AMNH), 3 9 , Jan. 27-Feb. 9, 1994, pitfalls, elev. $10 \mathrm{~m}$ (N. Platnick, K. Catley, R. Calderón, R. Allen, AMNH), 4 ․

DisTRIBUTION: Known only from Choapa and Petorca, Chile.

\section{Chilongius frayjorge, new species Figures 71-74}

TYPE: Male holotype taken at an elevation of $580 \mathrm{~m}$ in the relict Valdivian cloud-fed forest in the Parque Nacional Fray Jorge, Limarí, Región de Coquimbo (IV), Chile (Jan. 5, 1985; N. Platnick, O. Francke), deposited in $\mathrm{AMNH}$.

ETYMOLOGY: The specific name is a noun in apposition taken from the type locality.

DIAGNOSIS: Males can be distinguished by the distally very narrow embolus, which is restricted to the prolateral side of the palpal bulb (fig. 71), females by the relatively long, triangular epigynal atrium (figs. 73, 74).

MALE: Total length 3.37. Carapace 1.61 long, 1.32 wide. Femur II 1.30 long. Coloration as in C. palmas. Leg spination: femora: II p0-0-2; III d1-1-0; IV d1-1-0, r0-0-2; tibiae: I v2-2-2; II v1p-1p-2; IV d1-1-0, p1-1-
1; metatarsi: I v2-0-0; III p0-2-2; IV p1-2-1. Retrolateral tibial apophysis distally narrowed, tip rounded (fig. 72); embolus relatively short, restricted to prolateral side of bulb, greatly narrowed at tip (fig. 71).

FeMALE: Total length 4.11. Carapace 1.99 long, 1.50 wide. Femur II 1.40 long. Coloration as in C. palmas. Leg spination: femora: II p0-0-2; III r0-1-1; tibiae: II v2-2-0; III r01-1; IV d1-1-0; metatarsi: III p0-2-2. Epigynal atrium relatively long, triangular (fig. 73); paramedian epigynal ducts relatively wide (fig. 74).

Other Material Examined: CHILE: Región de Coquimbo (IV): Limarí: Parque Nacional Fray Jorge (relict Valdivian cloudfed forest), Oct. 3, 1992, elev. $560 \mathrm{~m}$ (N. Platnick, P. Goloboff, K. Catley, AMNH), 2\%, Feb. 9, pitfall (R. Calderón, AMNH), 10 .

Distribution: Known only from the cloud-fed forest at Fray Jorge in Coquimbo, Chile.

\section{Chilongius eltofo, new species} Figures 75-78

TYPE: Male holotype taken at an elevation of $80 \mathrm{~m}$ at La Herradure, Elqui, Región de 

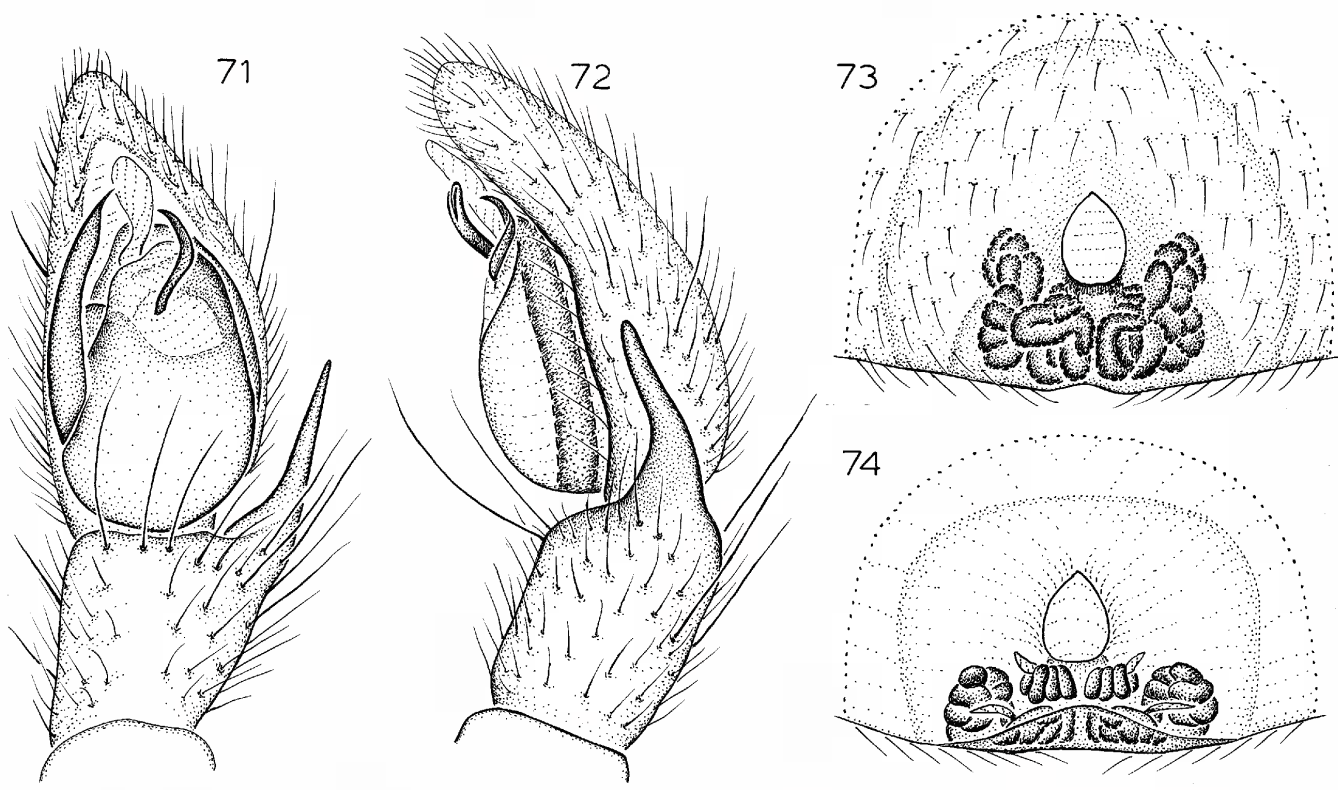

Figs. 71-74. Chilongius frayjorge, new species. 71. Left male palp, ventral view. 72. Same, retrolateral view. 73. Epigynum, ventral view. 74. Same, dorsal view.

Coquimbo (IV), Chile (Oct. 3, 1992; N. Platnick, P. Goloboff, K. Catley), deposited in AMNH.

ETYMOLOGY: The specific name is a noun in apposition taken from one of the localities at which the species has been collected.

DiAgNosis: Males can be distinguished by the wide, arrow-shaped tip of the embolus, the retrolateral edge of which is translucent (fig. 75), females by the medially invaginated posterior epigynal margin (figs. 77, 78).

MALE: Total length 5.48. Carapace 2.46 long, 1.98 wide. Femur II 1.69 long. Coloration as in C. palmas. Leg spination: femora: II d1-0-0; III r0-1-1; tibiae: I v2-2-1p; II v22-2; IV d1-1-0, p1-1-1; metatarsi: I v2-0-0; II v2-2-0; III p0-2-2, r0-1-2. Retrolateral tibial apophysis distally narrowed, tip rounded (fig. 76); embolus relatively short, very wide, retromarginal edge translucent (fig. 75).

FEMALE: Total length 4.58. Carapace 1.83 long, 1.46 wide. Femur II 1.31 long. Coloration as in C. palmas. Leg spination: femora: II p0-0-1; III r0-1-1; IV d1-1-0, r0-1-1; tibiae: III r0-1-1; IV p1-1-1; metatarsi: III p02-2. Epigynal atrium squared, posterior margin invaginated at midline (figs. 77, 78).
Other Material Examined: CHILE: Región de Coquimbo (IV): Elqui: beach $6 \mathrm{~km}$ S Cruz Grande, Oct. 7, 1992, elev. 20 m (N. Platnick, P. Goloboff, K. Catley, AMNH), 1 ; $16 \mathrm{~km} \mathrm{~S} \mathrm{Cruz} \mathrm{Grande,} \mathrm{Oct.} \mathrm{7,} \mathrm{1992,}$ elev. $140 \mathrm{~m}$ (N. Platnick, P. Goloboff, K. Catley, AMNH), 20, $1 \%$; $3 \mathrm{~km}$ E El Tofo, Jan. 7, 1985, under rocks, scrubby mountainside, elev. $45 \mathrm{~m}$ (N. Platnick, O. Francke, AMNH), 2q; La Herradura, Oct. 3, 1992, elev. $80 \mathrm{~m}$ (N. Platnick, P. Goloboff, K. Catley, AMNH), $1 \delta^{\hat{\sigma}} ; 20 \mathrm{~km} \mathrm{~N}$ La Serena, Route 5, km 491, $29^{\circ} 46^{\prime} \mathrm{S}, 71^{\circ} 20^{\prime} \mathrm{W}$, Oct. 67, 1992, elev. $120 \mathrm{~m}$ (N. Platnick, P. Goloboff, K. Catley, AMNH), 50 , Nov. 12, 1993, elev. 120 m (N. Platnick, K. Catley, M. Ramírez, R. Allen, AMNH), 1 ㅇ.

Distribution: Known only from Elqui, Chile.

Chilongius huasco, new species Figures 79-82

TYPE: Male holotype taken at an elevation of $170 \mathrm{~m}$ at a creek leading to Río Huasco, $25 \mathrm{~km}$ W Vallenar, Huasco, Región de Ata- 

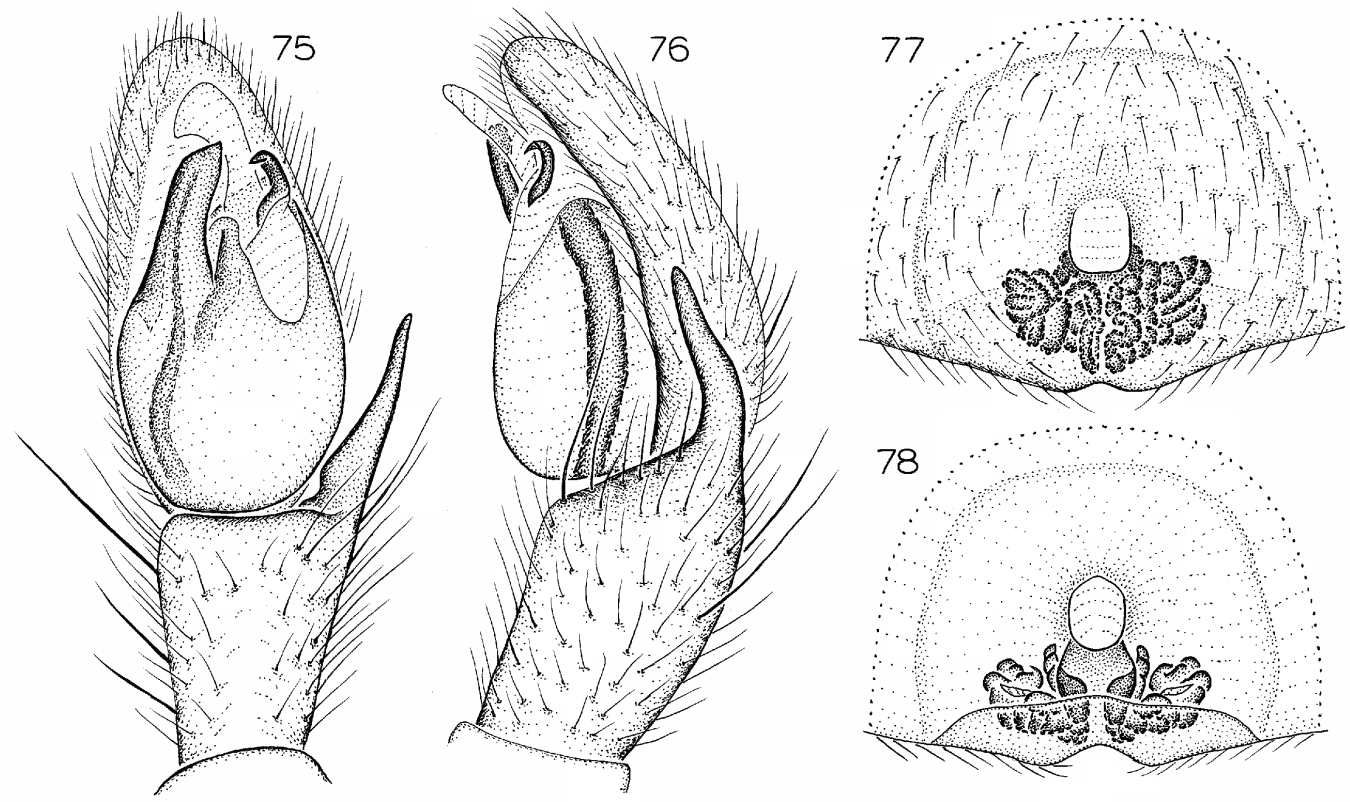

Figs. 75-78. Chilongius eltofo, new species. 75. Left male palp, ventral view. 76. Same, retrolateral view. 77. Epigynum, ventral view. 78. Same, dorsal view.

cama (III), Chile (Oct. 8, 1992; N. Platnick, P. Goloboff, K. Catley), deposited in AMNH.

ETYMOLOGY: The specific name is a noun in apposition taken from the type locality.

DiAgNosis: Males can be distinguished by the sharply bent tip of the embolus (fig. 79), females by the small, triangular epigynal atrium (fig. 81) and the anteriorly divergent paramedian epigynal ducts (fig. 82).

MALE: Total length 5.81. Carapace 2.64 long, 2.09 wide. Femur II 2.05 long. Coloration as in C. palmas. Leg spination: femora: I p0-1-1; II d1-0-0; III d1-1-0, r0-1-1; IV d11-0, r1-1-1; tibiae: I, II v2-2-2; III p1-1-1; IV d1-1-0, p1-1-1; metatarsi: I v2-0-0; III p1-22; IV p1-1-2. Retrolateral tibial apophysis distally narrowed, tip rounded (fig. 80); embolus sharply bent distally (fig. 79).

FEMALE: Total length 5.37. Carapace 1.97 long, 1.46 wide. Femur II missing. Coloration as in C. palmas. Leg spination (legs $\mathrm{I}$, II missing): femora: III d1-1-0; IV r0-0-1; tibiae: III r0-1-1; IV d1-1-0; metatarsi: III p1-2-2, r1-1-1; IV p1-2-1. Epigynal atrium relatively small, triangular (fig. 81); paramedian epigynal ducts anteriorly divergent (fig. 82).
Other Material Examined: CHILE: Región de Antofagasta (II): Antofagasta: $6 \mathrm{~km}$ E Paposo, Oct. 12, 1992, elev. $480 \mathrm{~m}(\mathrm{~N}$. Platnick, P. Goloboff, K. Catley, AMNH), 3 ô; 24 km S Paposo, Oct. 11, 1992, elev. 20-50 m (N. Platnick, P. Goloboff, K. Catley, AMNH), 1 to. Región de Atacama (III): Copiapó: Quebrada de León, N Caldera, Oct. 10, 1992, elev. $140 \mathrm{~m}$ (N. Platnick, P. Goloboff, K. Catley, AMNH), 1․ Huasco: creek leading to Río Huasco, $25 \mathrm{~km}$ W Vallenar, Oct. 8, 1992, elev. 170 m (N. Platnick, P. Goloboff, K. Catley, AMNH), 1 ๙ .

DISTRIBUTION: Known only from far northern Chile.

Chileuma, new genus

TyPe SPECIES: Chileuma paposo, new species.

Etymology: The generic name is a contraction of Chilean Theuma, male in gender.

Diagnosis: The combination of eight eyes and smooth tarsal claws separates members of this genus from the other Chilean theumines. Males can be recognized by the basally widened embolus (figs. 83, 87, 91), fe- 

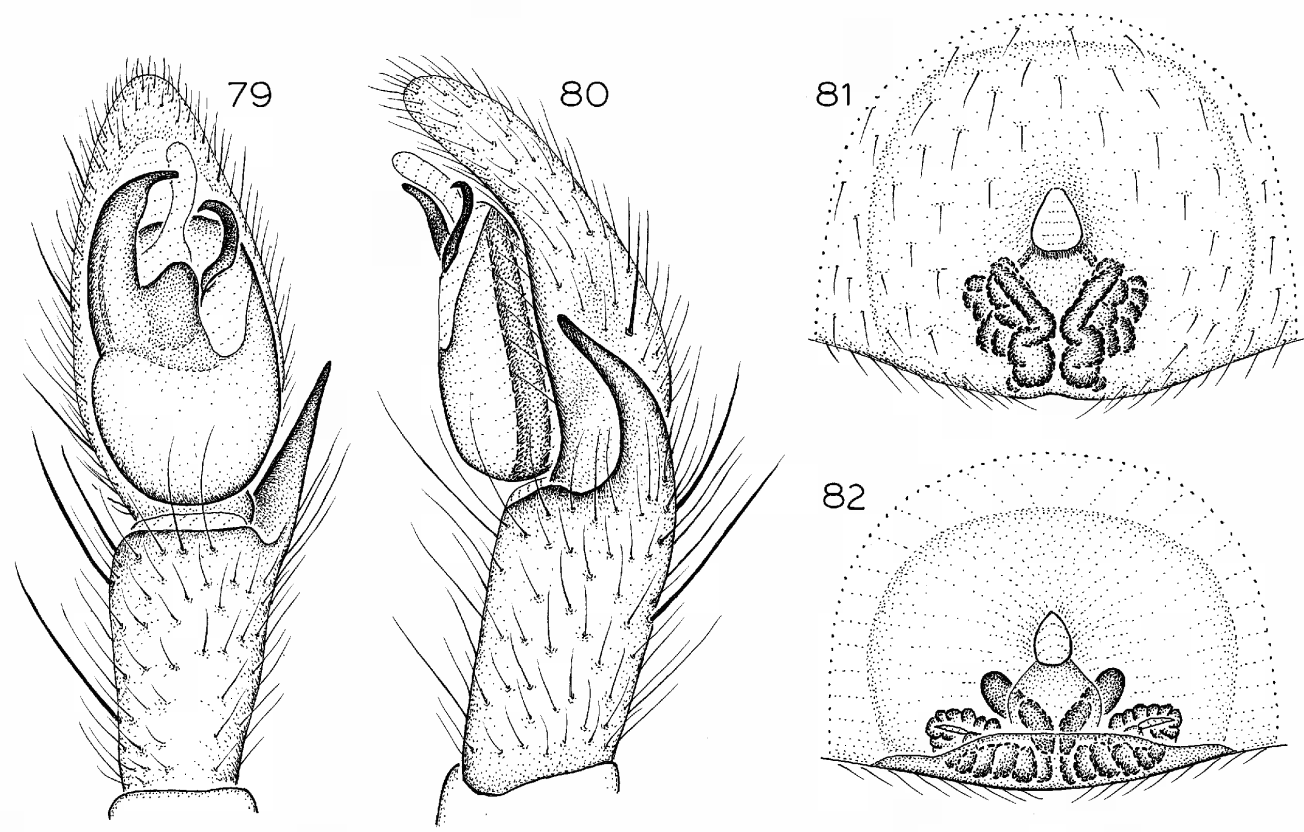

Figs. 79-82. Chilongius huasco, new species. 79. Left male palp, ventral view. 80. Same, retrolateral view. 81. Epigynum, ventral view. 82. Same, dorsal view.

males by the long, paramedian epigynal margins (figs. 85, 89, 93).

DESCRIPTION: Medium-sized spiders, total length 4.8-8.4. Carapace oval, narrowed in front to only about five-eighths its maximum width, with margins heavily sclerotized only at protruding anterolateral corners, posterior margin slightly invaginated at pedicel, slightly reflexed; pars thoracica coated with long, recumbent setae, without tubercles; thoracic groove long, shallow, longitudinal. Eight eyes in two rows, anterior medians smallest, circular, dark, anterior and posterior medians smaller than oval lateral pairs; posterior medians flat, irregularly rectangular; from above, both eye rows slightly recurved, from front, anterior row very slightly recurved, posterior row slightly procurved; anterior medians separated by slightly more than their diameter, closer to anterior laterals; posterior medians separated by about twice their width, about as far from posterior laterals; anterior and posterior laterals separated by about their width; median ocular quadrangle wider in back than in front, much wider in back than long. Chelicerae not projecting, slightly divergent, paturon with distinct, lightly sclerotized boss, promargin with row of long, curved setae, seta closest to fang base greatly elongated, distinctly bent toward midline at about one-eighth its length, many others on slight widening opposite base of fang; promargin with four widely spaced teeth, most proximal tooth smaller than others, retromargin with three widely spaced, tiny denticles; fang long, arched; chilum short, wide, unipartite. Labium slightly wider than long, widest at about one-fourth its length, posterolateral corners constricted; anterior margin strongly rebordered. Endites moderately long, convergent anteriorly, bent and obliquely depressed near anterior margin of labium; serrula present. Sternum shieldshaped, flat, with strongly rebordered lateral margins but rebordering abruptly absent along distinct posterior protrusion between coxae IV, not expanded anteriorly, with strong extensions to and smaller extensions between coxae; surface smooth, with short, erect setae, lateral margins and posterior protrusion with numerous long, erect setae, coxae IV widely separated. Single epimeric 

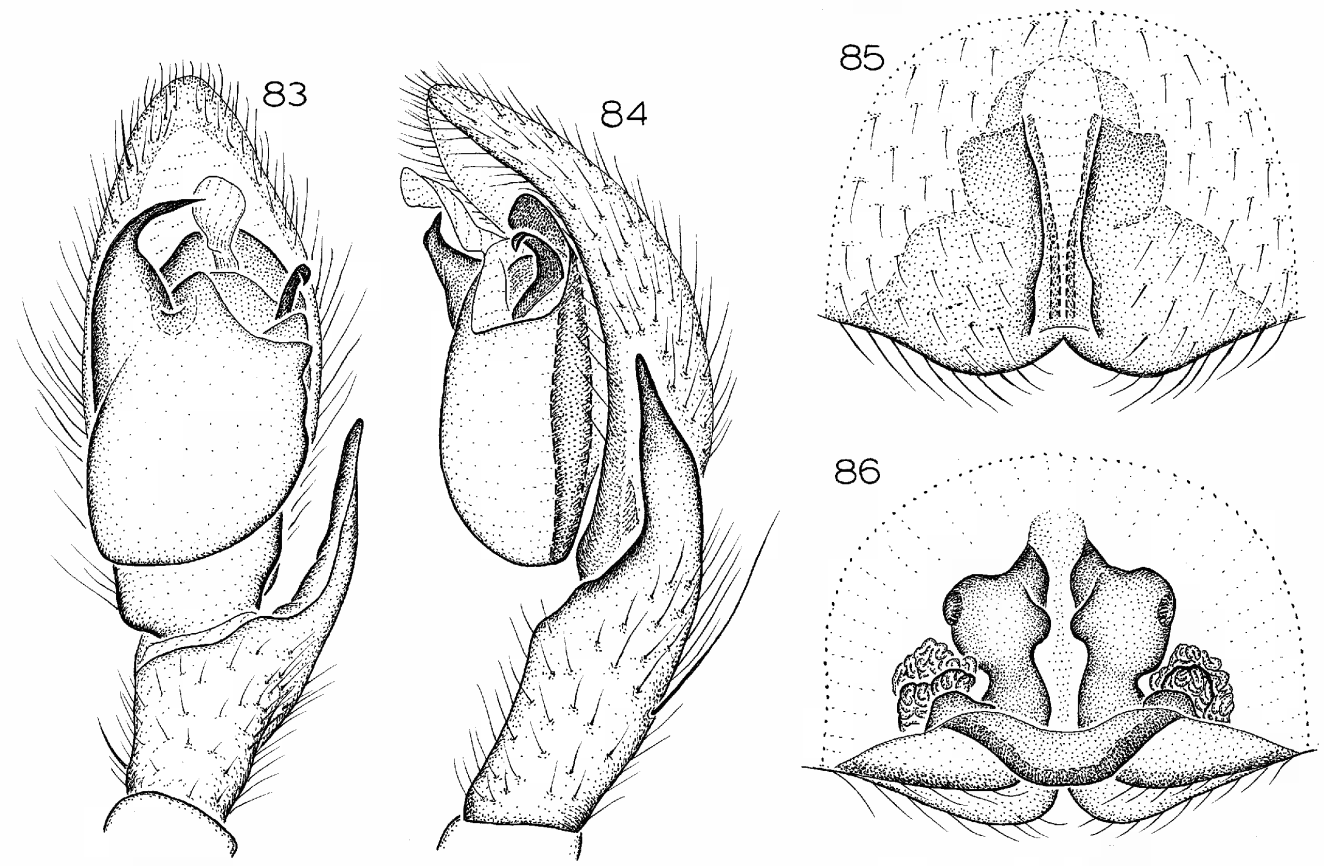

Figs. 83-86. Chileuma paposo, new species. 83. Left male palp, ventral view. 84. Same, retrolateral view. 85. Epigynum, ventral view. 86. Same, dorsal view.

sclerite on each side, above coxae, slightly extended between coxae, not fused to carapace. Pedicel composed of two dorsal sclerites (both sclerites excavated anteriorly) and sclerotized, triangular ventral sclerite adjacent to posterior tip of sternum.

Abdominal dorsum without anterior scutum in either sex, with two pairs of sigilla in anterior one-third of length; cuticle with long, recumbent setae, without clavate setae; epigastric scutum weakly sclerotized, without postepigastric sclerites, booklung covers not ridged; colulus absent, posterior spiracle situated well anterior of spinnerets, not as wide as separation between anterior lateral spinnerets. Six spinnerets, anterior laterals elongated, widely separated, slightly advanced anteriorly, with elongate ventral tubercle arising near cuticle of basal segment, bearing single major ampullate gland spigot, soft portions of cuticle bearing about nine piriform gland spigots with elongated bases, short shafts, and several accompanying setae (fig. 60); female posterior medians small, narrow, contiguous, with spigots closely clustered at tip, cluster apparently consisting of about seven large cylindrical gland spigots, one minor ampullate gland spigot with wide base, and around eight aciniform gland spigots with long, narrow shafts (fig. 61); female posterior laterals apparently with two large cylindrical gland spigots, single minor ampullate gland spigot, and many more distally situated aciniform gland spigots with smaller, narrower shafts (fig. 62).

Leg formula 4123 in females, 1423 in males, legs long, anterior pair greatly elongated in males, with erect, dark setae; coxae and trochanters without dorsal tubercles, fourth trochanters only slightly elongated; anterior coxae without protuberant posterolateral corners; trochanters not notched; anterior metatarsi and tarsi with ventral scopula very strong in females, much weaker in males; posterior metatarsi without distal preening brushes; tarsi without cuticular cracks, with two long claws lacking teeth, claw tufts each composed of about five distally widened setae; dorsal surface of tarsi with rebordered proximal margin; tricho- 
bothria in two rows dorsally on all tarsi and metatarsi. Female palpal femur, tibia, and tarsus with several long spines, tarsus long, not enlarged distally, without distodorsal pad of setae, with smooth claw. Leg spines of males slightly longer than those of females; typical leg spination pattern (only surfaces bearing spines listed): femora: I, II d1-1-0, p0-0-1; III d1-1-1, p0-1-1, r0-1-1; IV d1-1-1, p0-11, r0-0-1; tibiae: I v1p-0-0; II v2-2-1 p; III d01-0, p0-1-1, v2-2-2, r0-1-1; IV d1-1-0, p0-11, v2-2-2, r0-1-1; metatarsi: III p0-1-2, v2-22, r0-1-1; IV p1-1-2, v2-2-2, r1-2-1.

Male palpal tibia with long, strong, distally narrowed retrolateral apophysis; embolus wide basally, abruptly bent and narrowed distally, accompanied by transparent conductor; median apophysis relatively short, distally hooked. Epigynum with conspicuous paramedian margins; lateral ducts highly convoluted, confined to posterior half of epigynum.

\section{Chileuma paposo, new species Figures 83-86}

TyPES: Male holotype and female allotype taken at an elevation of $480 \mathrm{~m}$ at a site $6 \mathrm{~km}$ E of Paposo, Antofagasta, Región de Antofagasta (II), Chile (Oct. 12, 1992; N. Platnick, P. Goloboff, K. Catley), deposited in AMNH,

ETYMOLOGY: The specific name is a noun in apposition taken from the type locality.

DiAGNOSIS: Males (figs. 83, 84) lack the ventral denticle on the retrolateral tibial apophysis found in $C$. renca, and have the tip of that apophysis longer and more abruptly narrowed than that of $C$. serena (as well as a more angular embolar base); females of C. paposo (fig. 85) lack the anterior widening of the epigynal atrium found in the other two species.

Male: Total length 6.71. Carapace 3.17 long, 2.21 wide. Femur II 3.07 long. Carapace and mouthparts light brownish orange, sternum paler, abdomen beige, legs light yellow. Leg spination: femora: I, II p0-1-1; tibiae: I, II v2-2-2; metatarsi: I, II v2-2-2. Retrolateral tibial apophysis distally long, narrow (fig. 84); embolus relatively wide at base of bent portion (fig. 83).

FEMAle: Total length 8.36. Carapace 3.10 long, 2.42 wide. Femur II 2.72 long. Coloration as in male. Leg spination: femora: I, II p0-1-1; tibiae: II v1p-1p-1p; metatarsi: III p1-1-2. Epigynum with almost parallel lateral margins (fig. 85); convoluted lateral ducts relatively large, compact (fig. 86).

OTHER MATERIAL EXAMINED: CHILE: Región de Antofagasta (II): Tocopilla: Río Loa, $25 \mathrm{~km} \mathrm{~S}$ Quillagua, $21^{\circ} 54^{\prime} \mathrm{S}, 69^{\circ} 33^{\prime} \mathrm{W}$, Aug. 20, 1966 (E. Schlinger, M. Irwin, CAS), 1 ․ Antofagasta: Cerro Moreno, Aug. 8, 1972 (R. Calderón, AMNH), 1 q ; Las Vertientes, June 12, 1992 (L. Peña, AMNH), 1 ; ; 6 km E Paposo, Oct. 12, 1992, elev. 480 m (N. Platnick, P. Goloboff, K. Catley, AMNH), 4ô, 5ㅇ; 4 km N Paposo, Oct. 11, 1992, elev. 20-50 m (N. Platnick, P. Goloboff, K. Catley, AMNH), 1 ; Quebrada San Ramón, E Taltal, Feb. 6, 1942, elev. 2000 ft (J. Bird, AMNH), 1 ㅇ. Región de Atacama (III): Copiapó: Puerto Viejo, S Caldera, Oct. 15-16, 1992 (L. Peña, AMNH), 2 ; Quebrada de León, N Caldera, Oct. 10, 1992, elev. 140 m (N. Platnick, P. Goloboff, K. Catley, AMNH), 20, 1 오.

Distribution: Known only from the Antofagasta and Atacama regions of far northern Chile.

\section{Chileuma serena, new species} Figures 87-90

TYPE: Holotype male and allotype female taken at an elevation of $150 \mathrm{~m}$ at a site 19 $\mathrm{km} \mathrm{N}$ of La Serena, Elqui, Región de Coquimbo (IV), Chile (Nov. 1-4, 1981; N. Platnick, R. Schuh), deposited in AMNH.

ETyMology: The specific name is a noun in apposition taken from the type locality.

Diagnosis: Males (figs. 87, 88) lack the ventral denticle on the retrolateral tibial apophysis found in $C$. renca, and have the tip of that apophysis shorter and less abruptly narrowed than that of $C$. serena (as well as a less angular embolar base, especially obvious in retrolateral view); females resemble those of $C$. renca in having an anteriorly widened epigynal atrium (fig. 89), but the atrium is longer than in that species.

MALE: Total length 5.61. Carapace 2.43 long, 1.89 wide. Femur II 2.40 long. Coloration as in $C$. paposo. Leg spination: femora: I, II p0-1-1; III r0-0-1; IV r0-1-1; tibiae: I, II 

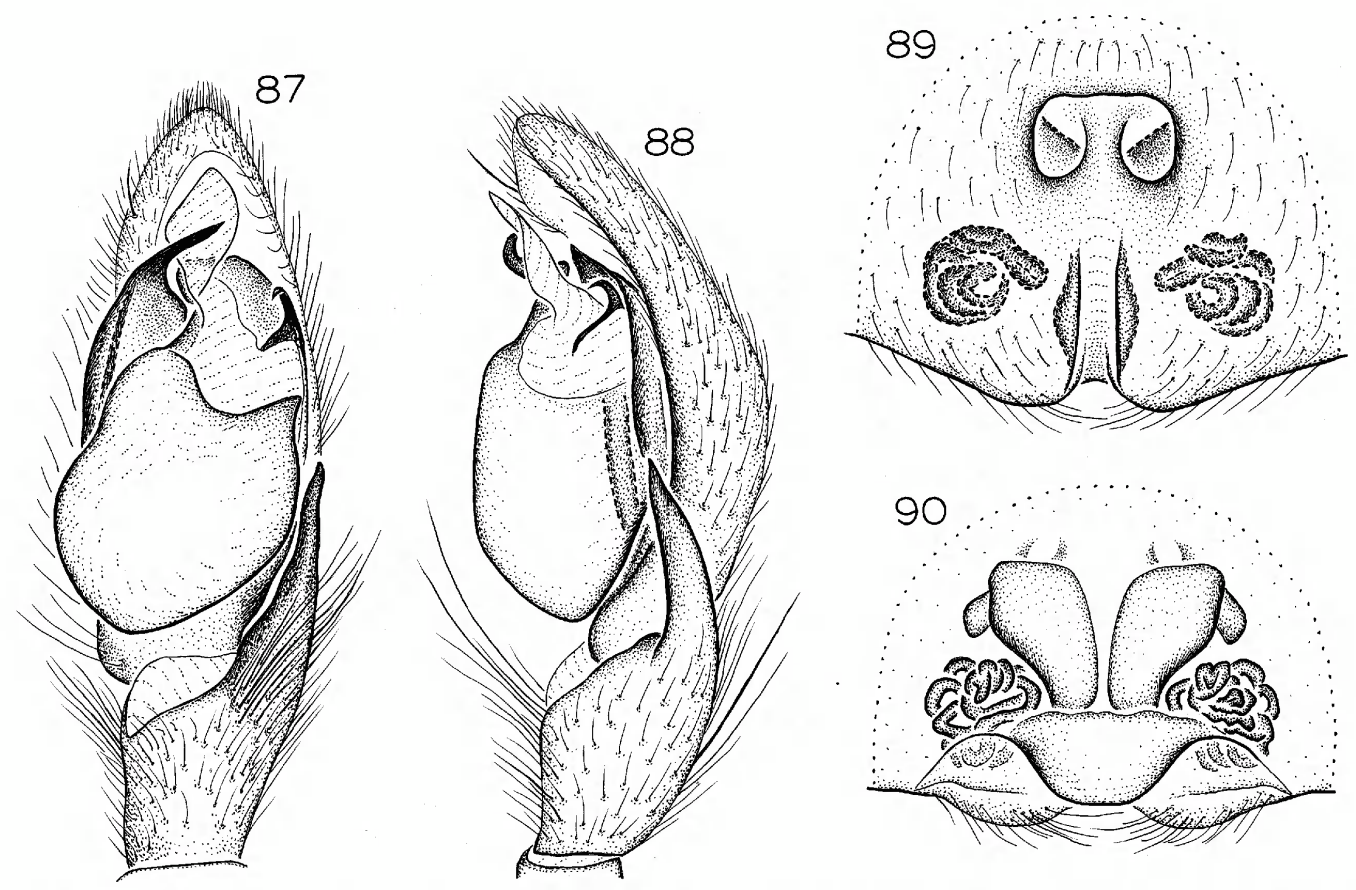

Figs. 87-90. Chileuma serena, new species. 87. Left male palp, ventral view. 88. Same, retrolateral view. 89. Epigynum, ventral view. 90. Same, dorsal view.

v2-2-2; metatarsi: I, II v2-2-0; III r0-2-1. Retrolateral tibial apophysis distally long, gradually narrowing distally (fig. 88); embolus relatively narrow at base of bent portion (fig. 87).

FEMALE: Total length 6.20. Carapace 3.03 long, 2.39 wide. Femur II 2.49 long. Coloration as in C. paposo. Leg spination: femora: II p0-1-1; tibiae: I v0-0-0; II v1p-1p-1p; metatarsi: III v2-0-2. Epigynum with anteriorly wide, rectangular atrium (fig. 89); convoluted lateral ducts forming wide mass (fig. 90).

Other Material Examined: CHILE: Región de Atacama (III): Huasco: Puerto Huasco, $28^{\circ} 28^{\prime} \mathrm{S}, 71^{\circ} 14^{\prime} \mathrm{W}$, July 2, 1966 (M. Irwin, N. Hichins, CAS), 1\%; Tongoy, W Freirina, Oct. 15, 1990 (L. Peña, AMNH), 1․ Región de Coquimbo (IV): Elqui: El Tofo, Sept. 1916 (AMNH), 2q; Cuesta Las Cardas, Aug. 3, 1961 (R. Wagenkneer, A. Archer, AMNH), 1\%; La Herradura, Oct. 3, 1992, elev. 80 m (N. Platnick, P. Goloboff, K. Catley, AMNH), 2q; 20 km N La Serena on Route $5, \mathrm{~km} 491,29^{\circ} 46^{\prime} \mathrm{S}, 71^{\circ} 20^{\prime} \mathrm{W}$, Oct. 7, 1992, elev. $120 \mathrm{~m}$ (N. Platnick, P. Goloboff, K. Catley, AMNH), 1q; 26 km S Vicuña, Nov. 10, 1992 (J. Rozen, AMNH), 1 ․ Limarí: Los Cristales, Ovalle, Sept. 1983 (L. Peña, AMNH), 49 ; Monte Patria, May 8-9, 1975 (L. Peña, AMNH), 1 ; Parque Nacional Fray Jorge (relict Valdivian cloud-fed forest), Aug. 10 (R. Calderón, AMNH), 1 \%. Choapa: Caleta Oscuro, Oct. 2, 1983 (E. Maury, MACN), 1q; $6.5 \mathrm{~km} \mathrm{~N}$ Los Vilos, Feb. 9, 1986, coastal dunes, elev. $10 \mathrm{~m}$ (N. Platnick, R. Schuh, AMNH), $1 \delta^{\hat{\sigma}}$, Oct. 16, 1992, same (N. Platnick, P. Goloboff, K. Catley, AMNH), 1 ㅇ.

Distribution: Known only from the Atacama and Coquimbo regions of northern Chile.

\section{Chileuma renca, new species} Figures 60-62, 91-94

TYPES: Male holotype and female allotype from Renca, Santiago, Región Metropolitana 

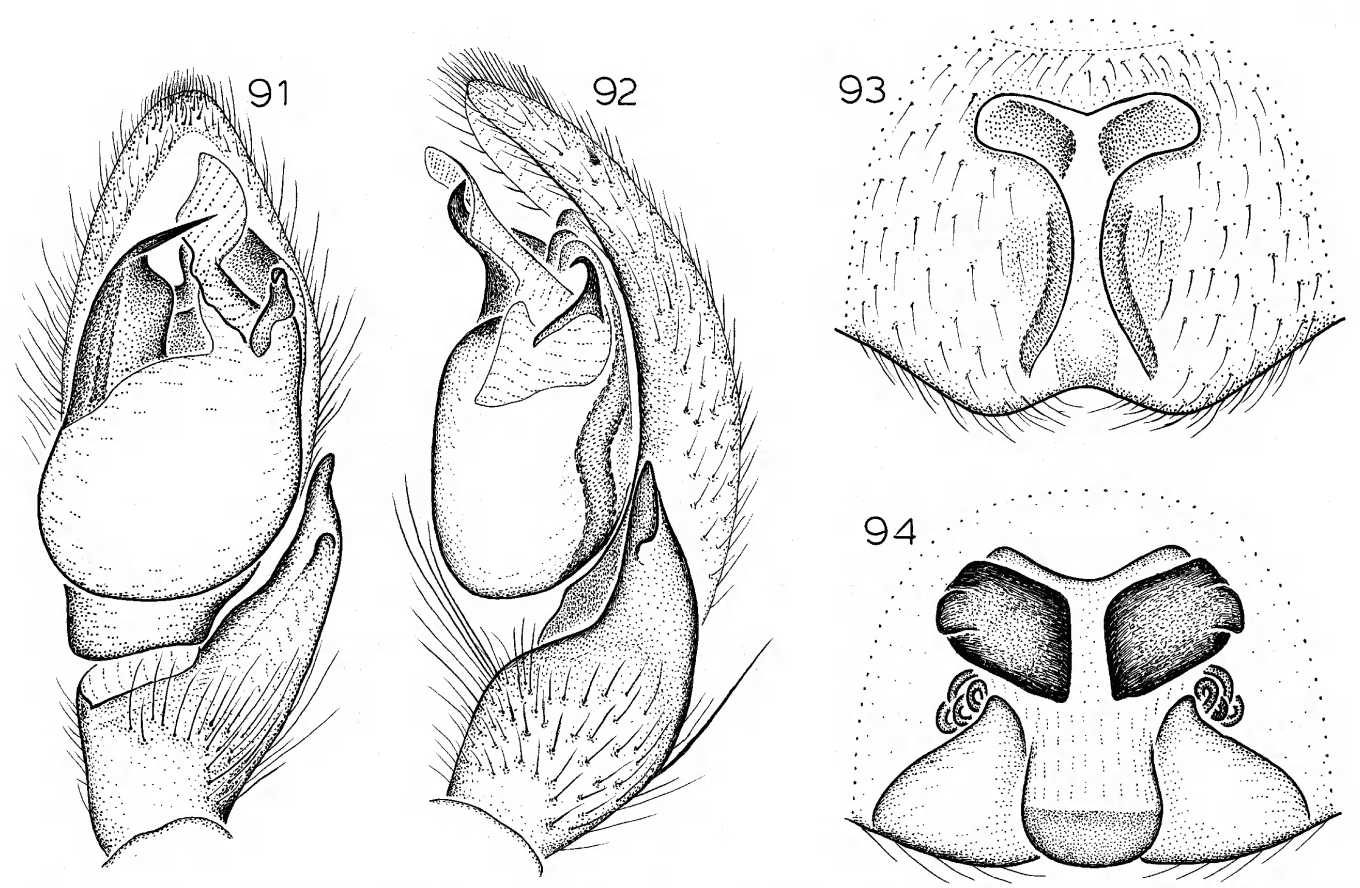

Figs. 91-94. Chileuma renca, new species. 91. Left male palp, ventral view. 92. Same, retrolateral view. 93. Epigynum, ventral view. 94. Same, dorsal view.

de Santiago, Chile (June 7-10, 1975; L. Peña), deposited in AMNH.

ETYMOLOGY: The specific name is a noun in apposition taken from the type locality.

DIAGNOSIS: Males can be recognized by the ventral denticle situated at about twothirds of the length of the retrolateral tibial apophysis (figs. 91, 92); females resemble those of $C$. serena in having an anteriorly widened epigynal atrium (fig. 93), but the atrium is shorter than in that species.

MALE: Total length 4.86. Carapace 2.26 long, 1.84 wide. Femur II 2.31 long. Coloration as in C. paposo. Leg spination: femora: I, II p0-1-1; III p0-2-1; tibiae: I, II v2-2-2; metatarsi: I, II v2-2-2; III p0-1-1, v2-0-2, r11-1. Retrolateral tibial apophysis with denticle on ventral edge at about two-thirds its length (fig. 92); embolar tip abruptly bent (fig. 91).

FEMALE: Total length 8.32. Carapace 2.86 long, 2.07 wide. Femur II 2.38 long. Coloration as in C. paposo. Leg spination: femora: I, II p0-1-1; IV r0-1-1; tibiae: I v0-0-0; II v1p-1p-1p; metatarsi: III v2-0-2. Epigynum with short but very wide anterior atrial expansion (fig. 93); convoluted lateral ducts relatively small (fig. 94).

Other Material Examined: CHILE: Región de Valparaíso (V): Petorca: Los Molles, Route 5, km 188, $32^{\circ} 14^{\prime} \mathrm{S}, 71^{\circ} 30^{\prime} \mathrm{W}$, Jan. 27, 1994, elev. 10 m (N. Platnick, K. Catley, R. Calderón, R. Allen, AMNH), $1 \delta^{\text {t; }}$ Quebrada del Chivato, $1 \mathrm{~km} \mathrm{~S}$ Los Molles, Oct. 2, 1992, elev. 10 m (N. Platnick, P. Goloboff, K. Catley, AMNH), 1 ․ San Felipe de Aconcagua: Parque Nacional La Campana, Feb. 11, 1986, dry hillside, elev. 980 m (N. Platnick, R. Calderón, R. Schuh, AMNH), 10 . Valparaíso: Río Marga Marga, Los Perales, $33^{\circ} 09^{\prime} \mathrm{S}, 71^{\circ} 19^{\prime} \mathrm{W}$, Oct. 13,1966 , elev. 330 m (M. Irwin, E. Schlinger, CAS), 2 ㅇ. Región Metropolitana de Santiago: Santiago: Renca, June 7-10, 1975 (L. Peña, AMNH), 10, 17ㅇ, Aug. 27, 1983 (L. Irarrazaval, AMNH), 2q; Quilicura, May 25, 1979 (L. Peña, AMNH), 3ㅇ, Aug.-Sept. 1979 (L. Peña, AMNH), 19 ㅇ. Region del O’Higgins 
(VI): Colchagua: Fundo La Pieza, Millahue, Cunaco, Mar. 25, 1961 (R. Donoso, AMNH), 1 ㅇ․

DistRIBUTION: Known only from central Chile.

\section{ACKNOWLEDGMENTS}

It is a pleasure to acknowledge the many contributions of the colleagues who helped with the field work for this project, over many years: Drs. Robert Allen, Raúl Calderón, Tomás Cekalovic, Oscar Francke, Pablo Goloboff, Luis Peña, Martín Ramírez, Randall Schuh, and Diana Silva. John Murphy and Michael Roberts provided helpful observations on the tarsal claws, spinnerets, and relationships of Moreno; Martín Ramírez and Barbara Baehr supplied very useful reviews of the draft manuscript. We also thank Drs. Charles Griswold (CAS), Cristina Scioscia (MACN), and Eduardo Arrozpide (MLP) for access to specimens in the collections they curate. Financial support for fieldwork from the Eppley Foundation for Reseach and the National Science Foundation is gratefully acknowledged.

\section{REFERENCES}

Cooke, J.A.L. 1964. A revisionary study of some spiders of the rare family Prodidomidae. Proceedings of the Zoological Society of London 142: 257-305.

Dalmas, R. de. 1919. Synopsis des araignées de la famille des Prodidomidae. Annales de la Société Entomologique de France 87: 279-340.

Gertsch, W.J., and N.I. Platnick. 1979. A revision of the spider family Mecicobothriidae (Araneae, Mygalomorphae). American Museum Novitates 2687: 1-32.
Mello-Leitão, C.F. de. 1940. Arañas de la provincia de Buenos Aires y de las gobernaciones de La Pampa, Neuquén, Río Negro y Chubut. Revista del Museo de La Plata (Nueva Serie, Sección Zoología) 2: 1-62.

Platnick, N.I. 1986. On the spider genus Oltacloea (Araneae, Gnaphosidae). Revue Arachnologique 7: 9-14.

Platnick, N.I. 1990. Spinneret morphology and the phylogeny of ground spiders (Araneae, Gnaphosidae). American Museum Novitates 2978: $1-42$.

Platnick, N.I. 2000. A relimitation and revision of the Australasian ground spider family Lamponidae (Araneae: Gnaphosoidea). Bulletin of the American Museum of Natural History 245: 1330.

Platnick, N.I. 2002. A revision of the Australasian ground spiders of the families Ammoxenidae, Cithaeronidae, Gallieniellidae, and Trochanteriidae (Araneae: Gnaphosoidea). Bulletin of the American Museum of Natural History 271: 1243.

Platnick, N.I., and B. Baehr. In press. A revision of the Australasian ground spiders of the family Prodidomidae (Araneae, Gnaphosoidea). Bulletin of the American Museum of Natural History.

Platnick, N.I., and H. Höfer. 1990. Systematics and ecology of ground spiders (Araneae, Gnaphosidae) from central Amazonian inundation forests. American Museum Novitates 2971: 116.

Platnick, N.I., and D. Penney. 2004. A revision of the widespread spider genus Zimiris (Araneae, Prodidomidae). American Museum Novitates 3450: 1-12.

Platnick, N.I., and M.U. Shadab. 1976. A revision of the spider genera Lygromma and Neozimiris (Araneae, Gnaphosidae). American Museum Novitates 2598: 1-23.

Roewer, C.F. 1955. Katalog der Araneen von 1758 bis 1940, bzw. 1954. Brussels, 2: 1-1751. 
Complete lists of all issues of the Novitates and the Bulletin are available at World Wide Web site http://library.amnh.org/pubs. Inquire about ordering printed copies via e-mail from scipubs@amnh.org or via standard mail from: American Museum of Natural History, LibraryScientific Publications, Central Park West at 79th St., New York, NY 10024. TEL: (212) 7695545. FAX: (212) 769-5009.

(2) This paper meets the requirements of ANSI/NISO Z39.48-1992 (Permanence of Paper). 